\title{
Modeling lifetime data with Weibull-Lindley distribution
}

\begin{abstract}
In this paper a new extension of the Lindley distribution is presented using the Weibull link function introduced and studied by Tahir et al., ${ }^{1}$ to develop a Weibull-Lindley distribution. We derive and discuss the mathematical and Statistical properties of the subject distribution along with its reliability analysis and inference for the parameters. Finally, the WeibullLindley distribution has been used to model four lifetime datasets and the results show that the proposed generalization performs better than the other known extensions of the Lindley distribution considered for the study.
\end{abstract}

Keywords: Lindley distribution, Weibull-Lindley distribution, mathematical properties, reliability function, parameter estimation, applications.
Volume 7 Issue 6 - 2018

leren TG, Oyamakin SO, Chukwu AU

Department of Statistics, University of Ibadan, Nigeria

Correspondence: leren TG, Department of Statistics, University of Ibadan, Ibadan, Nigeria,

Email ternagadfrey@gmail.com

Received: October 15, 2018 | Published: November 23, 2018

\section{Introduction}

The Lindley distribution introduced by Lindley et al., ${ }^{2}$ in the context of Bayesian analysis as a counter example of fiducial statistics, is defined by its probability density function (PDF) and cumulative distribution function $(\mathrm{CDF})$ as

$$
G(x)=1-\left[1+\frac{\theta x}{\theta+1}\right] e^{-\theta x}
$$

And

$$
\mathrm{g}(\mathrm{x})=\frac{\theta^{2}}{\theta+1}(1+\mathrm{x}) \mathrm{e}^{-\theta \mathrm{x}}
$$

respectively. For $\mathrm{x}>0, \theta>0$, where $\theta$ is the scale parameter of the Lindley distribution.

Details of this distribution, its mathematical and statistical properties, estimation of its parameter and application including the superiority of Lindley distribution over exponential distribution has been done by Ghitany et al., ${ }^{3}$ We have so many generalized families of distributions proposed by different researchers that are used in extending other distributions to produce compound distributions with better performance. These are several ways of adding one or more parameters to a distribution function which makes the resulting distribution richer and more flexible for modeling data. Abrief summary of some of these methods or families of distribution include the beta generalized family (Beta-G) by Eugene et al., ${ }^{4}$ the Kumaraswamy-G by Cordeiro et al. ${ }^{5}$ Transmuted family of distributions by Shaw et al., ${ }^{6}$ Gamma-G (type 1) by Zografos et al., ${ }^{7}$ McDonald-G by Alexander et al., ${ }^{8}$ Gamma-G (type 2 ) by Risti et al., ${ }^{9}$ Gamma-G (type 3 ) by Torabi et al., ${ }^{10}$ Log-gamma-G by Amini et al., ${ }^{9}$ Exponentiated T-X by Alzaghal et al., ${ }^{12}$ Exponentiated-G (EG) by Cordeiro et al., ${ }^{13}$ Logistic-G by Torabi et al., ${ }^{14}$ Gamma-X by Alzaatreh et al., ${ }^{15}$ Logistic-X by Tahir et al., ${ }^{16}$ Weibull-X by Alzaatreh et al., ${ }^{17}$ Weibull-G by Bourguignon et al., ${ }^{18}$ a new Weibull-G family by Tahir et al., ${ }^{1}$ a Lomax-G family by Cordeiro et al., ${ }^{19}$ a new generalized Weibull-G family by Cordeiro et al. ${ }^{20}$ and Beta Marshall-Olkin family of distributions by Alizadeh et al., ${ }^{21}$ and some other families of the distributions.

Hence, there are also some generalizations of the Lindley distribution recently proposed in the literature such as the transmuted
Lindley distribution by Merovci et al., ${ }^{23}$ the exponentiated Power Lindley distribution by Ashour et al., ${ }^{24}$ Generalized Lindley distribution by Nadarajah et al., ${ }^{24}$ Transmuted Generalized Lindley distribution by Elgarhy et al., ${ }^{25}$ Extended Power Lindley distribution by Alkarni et al., ${ }^{26}$ a two-parameter Lindley distribution by Shanker et al., ${ }^{27}$ the Lomax-Lindley distribution by Yahaya et al., ${ }^{28}$ Transmuted Two-Parameter Lindley distribution by Al-khazaleh et al.,29 and a three-parameter Lindley distribution by Shanker et al., ${ }^{30}$ The aim of this article is to introduce a new continuous distribution called Weibull-Lindley distribution $(W L n D)$ from the proposed family by Tahir et al., ${ }^{1}$ The remaining parts of this article are presented in sections as follows: We defined the new distribution and give its plots in section 2.1. Section 2.2 derived some properties of the new distribution. Section 2.3 proposes some reliability functions of the new distribution. The order statistics for the new distribution are also given in section 2.4. The maximum likelihood estimates (MLEs) of the unknown model parameters of the new distribution are obtained in section 2.5. In section 3 we carryout application of the proposed model with others to four lifetime datasets. Lastly, in section 4, we give the summary of our work and concluding remarks.

\section{Materials and methods}

\section{Construction of Weibull-Lindley distribution (WLnD)}

In the next section, we have defined the $c d f$ and $p d f$ of the WeibullLindley distribution $(W L n D)$ using the method proposed by Tahir et al. ${ }^{1}$ According to Tahir et al., ${ }^{1}$ the formula or Weibull link function for deriving the $c d f$ and $p d f$ of any Weibull-based continuous distribution is defined as:

$$
\mathrm{F}(\mathrm{x})=\int_{0}^{-\log [\mathrm{G}(\mathrm{x})]} \alpha \beta \mathrm{t}^{\beta-1} \mathrm{e}^{-\alpha \mathrm{t}^{\beta}} \mathrm{dt}=\mathrm{e}^{-\alpha\{-\log [\mathrm{G}(\mathrm{x})]\}^{\beta}}
$$

And

$$
\mathrm{f}(\mathrm{x})=\alpha \beta \frac{\mathrm{g}(\mathrm{x})}{\mathrm{G}(\mathrm{x})}\{-\log [\mathrm{G}(\mathrm{x})]\}^{\beta-1} \mathrm{e}^{-\alpha\{-\log [\mathrm{G}(\mathrm{x})]\}^{\beta}}
$$

respectively, where $\mathrm{g}(\mathrm{x})$ and $\mathrm{G}(\mathrm{x})$ are the $p d f$ and $c d f$ of any continuous distribution to be generalized respectively and $\alpha$ and $\beta$ are the two additional new parameters responsible for the shape of the distribution. 
Using equation (1.1) and (1.2) in (2.1.1) and (2.1.2) and simplifying, we obtain the $c d f$ and $p d f$ of the Weibull-Lindley random variable $X$ as:

$$
\mathrm{F}(\mathrm{x} ; \theta, \alpha, \beta)=\mathrm{e}^{-\alpha\left\{-\log \left[1-\left[1+\frac{\theta \mathrm{x}}{\theta+1}\right] \mathrm{e}^{-\theta \mathrm{x}}\right]\right\}^{\beta}}
$$

and

$$
\mathrm{f}(\mathrm{x} ; \theta, \alpha, \beta)=\frac{\alpha \beta \theta^{2}(1+\mathrm{x}) \mathrm{e}^{-\theta \mathrm{x}}}{(\theta+1)\left[1-\left[1+\frac{\theta \mathrm{x}}{\theta+1}\right] \mathrm{e}^{-\theta \mathrm{x}}\right]}\left\{-\log \left[1-\left[1+\frac{\theta \mathrm{x}}{\theta+1}\right] \mathrm{e}^{-\theta \mathrm{x}}\right]\right\}^{\beta-1} \mathrm{e}^{-\alpha\left\{-\log \left[1-\left[1+\frac{\theta \mathrm{x}}{\theta+1}\right] \mathrm{e}^{-\theta \mathrm{x}}\right]\right\}^{\beta}}
$$

respectively.

For $\mathrm{x}>0 ; \theta, \alpha, \beta>0$; where $\theta$ is a scale parameter and $\alpha$ and $\beta$ is a shape parameters of the Weibull-Lindley distribution.

The following is a graphical representation of the $p d f$ and $c d f$ of the Weibull-Lindley distribution. Given some values of the parameters $\alpha, \beta \& \theta$, we provide some possible graphs for the $p d f$ and the $c d f$ of the $W L n D$ as shown in Figure 1\&2 below: Figure 1 indicates that the $W L n D$ is a skewed distribution and can take various forms. This means that distribution can be very useful for datasets that are skewed.

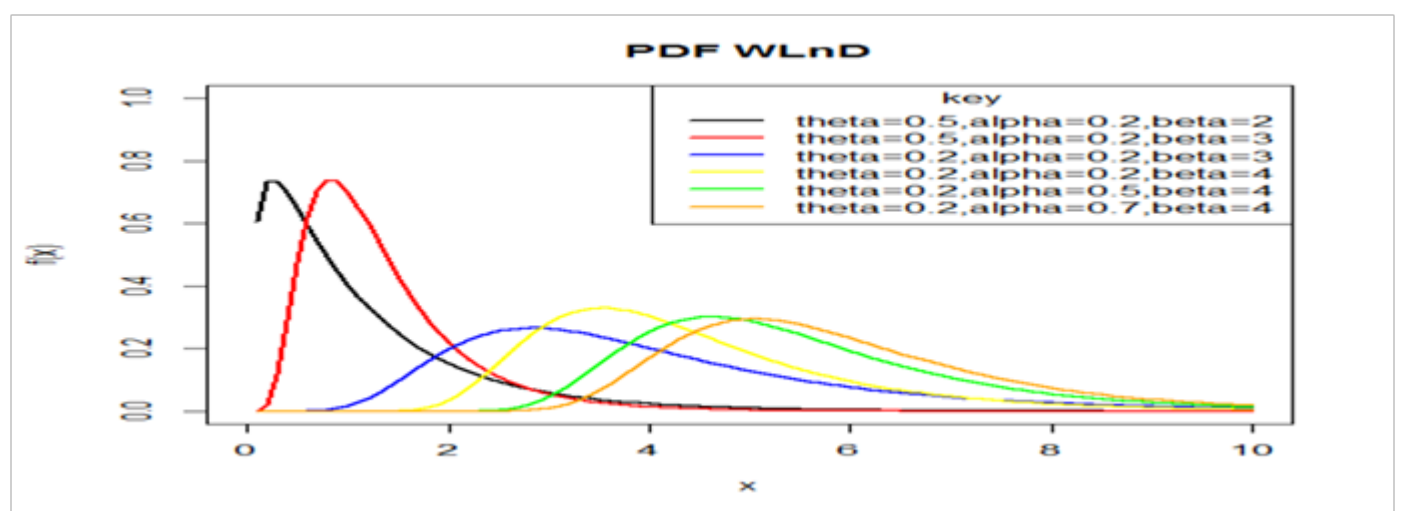

Figure I Graph of PDF of the WLnD for varrying parameter values.

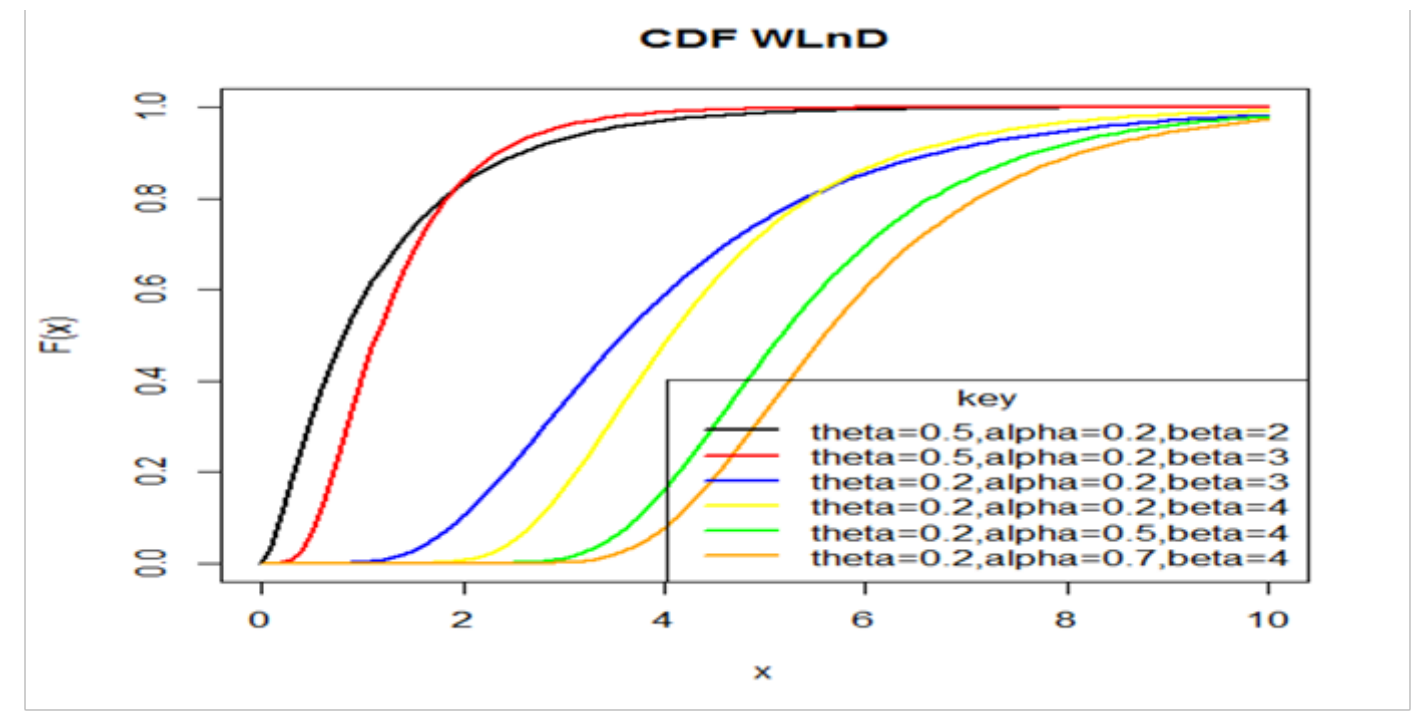

Figure 2 Graph of CDF of the WLnD for varying parameter values.

From the above $c d f$ plot, the $c d f$ increases when $X$ increases, and approaches 1 when $X$ becomes large, as expected.

\section{Properties}

In this section, we defined and discuss some properties of the $W L n D$ distribution.

\section{The Quantile function}

This function is derived by inverting the $c d f$ of any given continuous probability distribution. It is used for obtaining some moments like skewness and kurtosis as well as the median and for generation of random variables from the distribution in question. Hyndman et al., ${ }^{21}$ defined the quantile function for any distribution in the form $Q(u)=$ $F^{-1}(u)$ where $\mathrm{Q}(\mathrm{u})$ is the quantile function of $F(x)$ for $0<u<1$

Taking $F(x)$ to be the $c d f$ of the Weibull-Lindley distribution and inverting it as above will give us the Quantile function as follows:

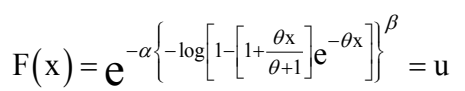


Simplifying equation (2.2.1) above, we obtain:

$$
\mathrm{Q}(\mathrm{u})=\mathrm{X}_{\mathrm{q}}=-1-\frac{1}{\theta}-\frac{1}{\theta} \mathrm{W}\left(-\frac{(\theta+1)\left(1-\exp \left\{-\left(-\frac{\ln \mathrm{u}}{\alpha}\right)^{\frac{1}{\alpha}}\right\}\right)}{\mathrm{e}^{\theta+1}}\right)
$$

By using (2.2.2) above, the median of $X$ from the $W L n D$ is simply obtained by setting $u=0.5$ while random numbers can be generated from $W L n D$ by setting $\mathrm{X}=\mathrm{Q}(\mathrm{u})$, where $u$ is a uniform variate on the unit interval $(0,1)$ and $\mathrm{W}($.) represents the negative branch of the Lambert function.

\section{Skewness and kurtosis}

The quantile based measures of skewness and kurtosis will employed due to non-existence of the classical measures in some cases. The Bowley's measure of skewness based on quartiles by Kenney et al., ${ }^{32}$ is given as;

$$
\mathrm{SK}=\frac{\mathrm{Q}\left(\frac{3}{4}\right)-2 \mathrm{Q}\left(\frac{1}{2}\right)+\mathrm{Q}\left(\frac{1}{4}\right)}{\mathrm{Q}\left(\frac{3}{4}\right)-\mathrm{Q}\left(\frac{1}{4}\right)}
$$

while the Risti et al.,9 kurtosis based on octiles is given by;

$$
\mathrm{KT}=\frac{\mathrm{Q}\left(\frac{7}{8}\right)-\mathrm{Q}\left(\frac{5}{8}\right)-\mathrm{Q}\left(\frac{3}{8}\right)+\left(\frac{1}{8}\right)}{\mathrm{Q}\left(\frac{6}{8}\right)-\mathrm{Q}\left(\frac{1}{4}\right)}
$$

where $Q($.$) is any quartile or octile of interest.$

\section{Moments}

Moments of a random variable are very important in distribution theory because they are used to study some of the most important features and characteristics of a random variable such as mean, variance, skewness and kurtosis.

Let $\mathrm{X}$ denote a continuous random variable, the $n^{\text {th }}$ moment of $\mathrm{X}$ is given by;

$$
\mu_{\mathrm{n}}^{\prime}=\mathrm{E}\left[\mathrm{X}^{\mathrm{n}}\right]=\int_{0}^{\infty} \mathrm{X}^{\mathrm{n}} \mathrm{f}(\mathrm{x}) \mathrm{dx}
$$

Considering $f(x)$ to be the $p d f$ of the Weibull-Lindley distribution as given in equation (2.1.4)

$$
\mu_{\mathrm{n}}^{\prime}=\mathrm{E}\left[\mathrm{X}^{\mathrm{n}}\right]=\int_{0}^{1} \mathrm{X}^{\mathrm{n}} \mathrm{f}(\mathrm{x}) \mathrm{dx}
$$

Recall that from equation (2.1.4),

$$
\mathrm{f}(\mathrm{x} ; \theta, \alpha, \beta)=\frac{\alpha \beta \theta^{2}(1+\mathrm{x}) \mathrm{e}^{-\theta \mathrm{x}}}{(\theta+1)\left[1-\left[1+\frac{\theta \mathrm{x}}{\theta+1}\right] \mathrm{e}^{-\theta \mathrm{x}}\right]}\left\{-\log \left[1-\left[1+\frac{\theta \mathrm{x}}{\theta+1}\right] \mathrm{e}^{-\theta \mathrm{x}}\right]\right\}^{\beta-1} \mathrm{e}^{-\alpha\left\{-\log \left[1-\left[1+\frac{\theta \mathrm{x}}{\theta+1}\right] \mathrm{e}^{-\theta \mathrm{x}}\right]\right\}^{\beta}}
$$

Let

$$
\mathrm{A}=\mathrm{e}^{-\alpha\left\{-\log \left[1-\left[1+\frac{\theta \mathrm{x}}{\theta+1}\right] \mathrm{e}^{-\theta \mathrm{x}}\right]\right\}^{\beta}}
$$

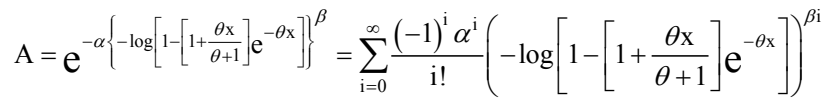

Then, using a power series expansiob for A,we can write $\mathrm{A}$ as:

Substituting for the expansion above in equation (2.2.6), we have;

$$
\begin{aligned}
& \mathrm{f}(\mathrm{x})=\frac{\alpha \beta \theta^{2}(1+\mathrm{x}) \mathrm{e}^{-\theta \mathrm{x}}}{(\theta+1)\left[1-\left[1+\frac{\theta \mathrm{x}}{\theta+1}\right] \mathrm{e}^{-\theta \mathrm{x}}\right]}\left\{-\log \left[1-\left[1+\frac{\theta \mathrm{x}}{\theta+1}\right] \mathrm{e}^{-\theta \mathrm{x}}\right]\right\}^{\beta-1} \sum_{\mathrm{i}=0}^{\infty} \frac{(-1)^{\mathrm{i}} \alpha^{\mathrm{i}}}{\mathrm{i} !}\left(-\log \left[1-\left[1+\frac{\theta \mathrm{x}}{\theta+1}\right] \mathrm{e}^{-\theta \mathrm{x}}\right]\right)^{\beta \mathrm{i}} \\
& \mathrm{f}(\mathrm{x})=\frac{\alpha \beta \theta^{2}(1+\mathrm{x}) \mathrm{e}^{-\theta \mathrm{x}}}{(\theta+1)\left[1-\left[1+\frac{\theta \mathrm{x}}{\theta+1}\right] \mathrm{e}^{-\theta \mathrm{x}}\right]} \sum_{\mathrm{i}=0}^{\infty} \frac{(-1)^{\mathrm{i}} \alpha^{\mathrm{i}}}{\mathrm{i} !}\left(-\log \left[1-\left[1+\frac{\theta \mathrm{x}}{\theta+1}\right] \mathrm{e}^{-\theta \mathrm{x}}\right]\right)^{\beta(\mathrm{i}+1)-1}
\end{aligned}
$$

Also, let

$$
\mathrm{B}=\left(-\log \left[1-\left[1+\frac{\theta \mathrm{x}}{\theta+1}\right] \mathrm{e}^{-\theta \mathrm{x}}\right]\right)^{\beta(\mathrm{i}+1)-1}
$$

Now, considering the following formula from Tahir et al., ${ }^{1}$ which holds for $\mathrm{B}$ for $\mathrm{i} \geq 1$, and then we can write $\mathrm{B}$ as follows:

$$
\left(-\log \left[1-\left[1+\frac{\theta \mathrm{x}}{\theta+1}\right] \mathrm{e}^{-\theta \mathrm{x}}\right]\right)^{\beta(\mathrm{i}+1)-1}=\sum_{\mathrm{k}, \mathrm{l}=0}^{\infty} \sum_{\mathrm{j}=0}^{\mathrm{k}} \frac{(-1)^{\mathrm{j}+\mathrm{k}+1}(\beta(\mathrm{i}+1))}{(\beta(\mathrm{i}+1)-1-\mathrm{j})}\left(\begin{array}{c}
\mathrm{k}-(\beta(\mathrm{i}+1)-1) \\
\mathrm{k}
\end{array}\right)\left(\begin{array}{c}
\mathrm{k} \\
\mathrm{j}
\end{array}\right)\left(\begin{array}{c}
(\beta(\mathrm{i}+1)-1)+\mathrm{k} \\
1
\end{array}\right) \mathrm{P}_{\mathrm{j}, \mathrm{k}}\left[1-\left[1+\frac{\theta \mathrm{x}}{\theta+1}\right] \mathrm{e}^{-\theta \mathrm{x}}\right]^{1}
$$

Where for (for $\mathrm{j} \geq 0$ ) $\mathrm{P}_{\mathrm{j}, 0}=1$ and (for $\mathrm{k}=1,2, \ldots \ldots$ ) 
$\mathrm{P}_{\mathrm{j}, \mathrm{k}}=\mathrm{k}^{-1} \sum_{\mathrm{m}=1}^{\mathrm{k}}(-1)^{\mathrm{m}} \frac{[\mathrm{m}(\mathrm{j}+1)-\mathrm{k}]}{(\mathrm{m}+1)} \mathrm{P}_{\mathrm{j}, \mathrm{k}-\mathrm{m}}$
Combining equation (2.2.8) and (2.2.9) and inserting the above power series in equation (2.2.7) and simplifying, we have:

$$
\begin{aligned}
& \mathrm{f}(\mathrm{x})=\frac{\alpha \beta \theta^{2}}{(\theta+1)} \sum_{\mathrm{i}=0}^{\infty} \frac{(-1)^{\mathrm{i}} \alpha^{\mathrm{i}}}{\mathrm{i} !} \sum_{\mathrm{k}, \mathrm{l}=0}^{\infty} \sum_{\mathrm{j}=0}^{\mathrm{k}} \frac{(-1)^{\mathrm{j}+\mathrm{k}+1}(\beta(\mathrm{i}+1))}{(\beta(\mathrm{i}+1)-1-\mathrm{j})}\left(\begin{array}{c}
\mathrm{k}-(\beta(\mathrm{i}+1)-1) \\
\mathrm{k}
\end{array}\right)\left(\begin{array}{c}
\mathrm{k} \\
\mathrm{j}
\end{array}\right)\left(\begin{array}{c}
(\beta(\mathrm{i}+1)-1)+\mathrm{k} \\
1
\end{array}\right) \mathrm{P}_{\mathrm{j}, \mathrm{k}}(1+\mathrm{x}) \mathrm{e}^{-\theta \mathrm{x}}\left[1-\left[1+\frac{\theta \mathrm{x}}{\theta+1}\right] \mathrm{e}^{-\theta \mathrm{x}}\right]^{1-1} \\
& \mathrm{f}(\mathrm{x})=\frac{\alpha \beta \theta^{2}}{(\theta+1)} \sum_{\mathrm{i}=0}^{\infty} \sum_{\mathrm{k}, \mathrm{l}=0}^{\infty} \sum_{\mathrm{j}=0}^{\mathrm{k}} \frac{(-1)^{\mathrm{i}+\mathrm{j}+\mathrm{k}+1} \alpha^{\mathrm{i}+1}(\beta(\mathrm{i}+1))}{\mathrm{i} !(\beta(\mathrm{i}+1)-1-\mathrm{j})}\left(\begin{array}{c}
\mathrm{k}-(\beta(\mathrm{i}+1)-1) \\
\mathrm{k}
\end{array}\right)\left(\begin{array}{c}
\mathrm{k} \\
\mathrm{j}
\end{array}\right)\left(\begin{array}{c}
(\beta(\mathrm{i}+1)-1)+\mathrm{k} \\
1
\end{array}\right) \mathrm{P}_{\mathrm{j}, \mathrm{k}}(1+\mathrm{x}) \mathrm{e}^{-\theta \mathrm{x}}\left[1-\left[1+\frac{\theta \mathrm{x}}{\theta+1}\right] \mathrm{e}^{-\theta \mathrm{x}}\right]^{1-1}
\end{aligned}
$$

Now,if 1 is a positive non-integer, we can expand the last term in (2.2.10) as:

$$
\left[1-\left[1+\frac{\theta \mathrm{x}}{\theta+1}\right] \mathrm{e}^{-\theta \mathrm{x}}\right]^{1-1}=\sum_{\mathrm{m}=0}^{\infty}(-1)^{\mathrm{m}}\left(\begin{array}{c}
1-1 \\
\mathrm{~m}
\end{array}\right)\left[\left[1+\frac{\theta \mathrm{x}}{\theta+1}\right] \mathrm{e}^{-\theta \mathrm{x}}\right]^{\mathrm{m}}
$$

Therefore, $\mathrm{f}(\mathrm{x})$ becomes:

$$
\mathrm{f}(\mathrm{x})=\frac{\alpha \beta \theta^{2}}{(\theta+1)} \sum_{\mathrm{m}=0}^{\infty} \sum_{\mathrm{i}=0}^{\infty} \sum_{\mathrm{k}, \mathrm{l}=0}^{\infty} \sum_{\mathrm{j}=0}^{\mathrm{k}} \frac{(-1)^{\mathrm{i}+\mathrm{j}+\mathrm{k}+1+\mathrm{m}} \alpha^{\mathrm{i}+1}(\beta(\mathrm{i}+1))}{\mathrm{i} !(\beta(\mathrm{i}+1)-1-\mathrm{j})}\left(\begin{array}{c}
\mathrm{k}-(\beta(\mathrm{i}+1)-1) \\
\mathrm{k}
\end{array}\right)\left(\begin{array}{c}
1-1 \\
\mathrm{~m}
\end{array}\right)\left(\begin{array}{c}
\mathrm{k} \\
\mathrm{j}
\end{array}\right)\left(\begin{array}{c}
(\beta(\mathrm{i}+1)-1)+\mathrm{k} \\
1
\end{array}\right) \mathrm{P}_{\mathrm{j}, \mathrm{k}}(1+\mathrm{x}) \mathrm{e}^{-\theta \mathrm{x}(1+\mathrm{m})}\left[1+\frac{\theta \mathrm{x}}{\theta+1}\right]^{\mathrm{m}}
$$

Using power series expansion on the last term in equation (2.2.12), we have

$\left(1+\frac{\theta \mathrm{x}}{\theta+1}\right)^{\mathrm{m}}=\sum_{\mathrm{p}=0}^{\infty}\left(\begin{array}{c}\mathrm{m} \\ \mathrm{p}\end{array}\right)\left(\frac{\theta \mathrm{x}}{\theta+1}\right)^{\mathrm{p}}=\sum_{\mathrm{p}=0}^{\infty}\left(\begin{array}{c}\mathrm{m} \\ \mathrm{p}\end{array}\right)\left(\frac{\theta}{\theta+1}\right)^{\mathrm{p}} \mathrm{x}^{\mathrm{p}}$

Now, substituting equation (2.2.13), the power series expansion in equation (2.2.12) above, one gets:

$\mathrm{f}(\mathrm{x})=\frac{\alpha \beta \theta^{2}}{(\theta+1)} \sum_{\mathrm{p}=0}^{\infty} \sum_{\mathrm{m}=0}^{\infty} \sum_{\mathrm{i}=0}^{\infty} \sum_{\mathrm{k}, \mathrm{l}=0}^{\infty} \sum_{\mathrm{j}=0}^{\mathrm{k}} \frac{(-1)^{\mathrm{i}+\mathrm{j}+\mathrm{k}+1+\mathrm{m}} \alpha^{\mathrm{i}+1}(\beta(\mathrm{i}+1))}{\mathrm{i} !(\beta(\mathrm{i}+1)-1-\mathrm{j})}\left(\begin{array}{c}\mathrm{k}-(\beta(\mathrm{i}+1)-1) \\ \mathrm{k}\end{array}\right)\left(\begin{array}{c}1-1 \\ \mathrm{~m}\end{array}\right)\left(\begin{array}{c}\mathrm{m} \\ \mathrm{p}\end{array}\right)\left(\begin{array}{c}\theta \\ \theta+1\end{array}\right)^{\mathrm{p}}\left(\begin{array}{c}\mathrm{k} \\ \mathrm{j}\end{array}\right)\left(\begin{array}{c}(\beta(\mathrm{i}+1)-1)+\mathrm{k} \\ 1\end{array}\right) \mathrm{P}_{\mathrm{j}, \mathrm{k}}(1+\mathrm{x}) \mathrm{x}^{\mathrm{p}} \mathrm{e}^{-\theta \mathrm{x}(1+\mathrm{m})}$

This implies that:

$\mathrm{f}(\mathrm{x})=\eta_{\mathrm{i}, \mathrm{j}, \mathrm{k}, \mathrm{l}, \mathrm{m}, \mathrm{p}}(1+\mathrm{x}) \mathrm{x}^{\mathrm{p}} \mathrm{e}^{-\theta \mathrm{x}(1+\mathrm{m})}$

Where

$\eta_{\mathrm{i}, \mathrm{j}, \mathrm{k}, \mathrm{l}, \mathrm{m}, \mathrm{p}}=\frac{\alpha \beta \theta^{2}}{(\theta+1)} \sum_{\mathrm{p}=0}^{\infty} \sum_{\mathrm{m}=0}^{\infty} \sum_{\mathrm{i}=0}^{\infty} \sum_{\mathrm{k}, 1=0}^{\infty} \sum_{\mathrm{j}=0}^{\mathrm{k}} \frac{(-1)^{\mathrm{i}+\mathrm{j}+\mathrm{k}+\mathrm{l}+\mathrm{m}} \alpha^{\mathrm{i}+1}(\beta(\mathrm{i}+1))}{\mathrm{i} !(\beta(\mathrm{i}+1)-1-\mathrm{j})}\left(\begin{array}{c}\mathrm{k}-(\beta(\mathrm{i}+1)-1) \\ \mathrm{k}\end{array}\right)\left(\begin{array}{c}1-1 \\ \mathrm{~m}\end{array}\right)\left(\begin{array}{c}\mathrm{m} \\ \mathrm{p}\end{array}\right)\left(\frac{\theta}{\theta+1}\right)^{\mathrm{p}}\left(\begin{array}{c}\mathrm{k} \\ \mathrm{j}\end{array}\right)\left(\begin{array}{c}(\beta(\mathrm{i}+1)-1)+\mathrm{k} \\ 1\end{array}\right) \mathrm{P}_{\mathrm{j}, \mathrm{k}}$

Hence,

$\mu_{\mathrm{n}}^{\prime}=\mathrm{E}\left[\mathrm{X}^{\mathrm{n}}\right]=\int_{0}^{\infty} \mathrm{X}^{\mathrm{n}} \mathrm{f}(\mathrm{x}) \mathrm{dx}=\int_{0}^{\infty} \eta_{\mathrm{i}, \mathrm{j}, \mathrm{k}, 1, \mathrm{~m}, \mathrm{p}}(1+\mathrm{x}) \mathrm{x}^{\mathrm{n}+\mathrm{p}} \mathrm{e}^{-\theta \mathrm{x}(1+\mathrm{m})} \mathrm{dx}$

$\mu_{\mathrm{n}}^{\prime}=\mathrm{E}\left[\mathrm{X}^{\mathrm{n}}\right]=\int_{0}^{\infty} \mathrm{X}^{\mathrm{n}} \mathrm{f}(\mathrm{x}) \mathrm{dx}=\eta_{\mathrm{i}, \mathrm{j}, \mathrm{k}, 1, \mathrm{~m}, \mathrm{p}}\left[\int_{0}^{\infty} \mathrm{x}^{\mathrm{n}+\mathrm{p}} \mathrm{e}^{-\theta \mathrm{x}(1+\mathrm{m})} \mathrm{dx}+\int_{0}^{\infty} \mathrm{x}^{\mathrm{n}+\mathrm{p}+1} \mathrm{e}^{-\theta \mathrm{x}(1+\mathrm{m})} \mathrm{dx}\right]$

Also, using integration by substitution method in equation (2.2.15); we obtain the following:

Let $\mathrm{u}=\theta \mathrm{x}(1+\mathrm{m}) \Rightarrow \mathrm{x}=\frac{\mathrm{u}}{\theta(1+\mathrm{m})} ; \frac{\mathrm{du}}{\mathrm{dx}}=\theta(1+\mathrm{m})$ and $\mathrm{dx}=\frac{\mathrm{du}}{\theta(1+\mathrm{m})}$

Substituting for $\mathrm{u}, \mathrm{x}$ and $\mathrm{dx}$ in equation (2.2.15) and simplifying; we have:

$$
\begin{aligned}
& \mu_{\mathrm{n}}^{\prime}=\eta_{\mathrm{i}, \mathrm{j}, \mathrm{k}, \mathrm{l}, \mathrm{m}, \mathrm{p}}\left[\int_{0}^{\infty}\left(\frac{\mathrm{u}}{\theta(1+\mathrm{m})}\right)^{\mathrm{n}+\mathrm{p}} \mathrm{e}^{-\mathrm{u}}\left(\frac{\mathrm{du}}{\theta(1+\mathrm{m})}\right)+\int_{0}^{\infty}\left(\frac{\mathrm{u}}{\theta(1+\mathrm{m})}\right)^{\mathrm{n}+\mathrm{p}+1} \mathrm{e}^{-\mathrm{u}}\left(\frac{\mathrm{du}}{\theta(1+\mathrm{m})}\right)\right] \\
& \mu_{\mathrm{n}}^{\prime}=\eta_{\mathrm{i}, \mathrm{j}, \mathrm{k}, \mathrm{l}, \mathrm{m}, \mathrm{p}}\left[\frac{1}{(\theta(1+\mathrm{m}))^{\mathrm{n}+\mathrm{p}+1}} \int_{0}^{\infty} \mathrm{u}^{\mathrm{n}+\mathrm{p}} \mathrm{e}^{-\mathrm{u}} \mathrm{du}+\frac{1}{(\theta(1+\mathrm{m}))^{\mathrm{n}+\mathrm{p}+2}} \int_{0}^{\infty} \mathrm{u}^{\mathrm{n}+\mathrm{p}+1} \mathrm{e}^{-\mathrm{u}} \mathrm{du}\right]
\end{aligned}
$$




$$
\mu_{\mathrm{n}}^{\prime}=\eta_{\mathrm{i}, \mathrm{j}, \mathrm{k}, \mathrm{l}, \mathrm{m}, \mathrm{p}}\left[\frac{1}{(\theta(1+\mathrm{m}))^{\mathrm{n}+\mathrm{p}+1}} \int_{0}^{\infty} \mathrm{u}^{\mathrm{n}+\mathrm{p}+1-1} \mathrm{e}^{-\mathrm{u}} \mathrm{du}+\frac{1}{(\theta(1+\mathrm{m}))^{\mathrm{n}+\mathrm{p}+2}} \int_{0}^{\infty} \mathrm{u}^{\mathrm{n}+\mathrm{p}+2-1} \mathrm{e}^{-\mathrm{u}} \mathrm{du}\right]
$$

$$
\begin{aligned}
& \text { Again recall that } \int_{0}^{\infty} \mathrm{t}^{\mathrm{k}-1} \mathrm{e}^{-\mathrm{t}} \mathrm{dt}=\Gamma(\mathrm{k}) \text { and that } \\
& \int_{0}^{\infty} \mathrm{t}^{\mathrm{k}} \mathrm{e}^{-\mathrm{t}} \mathrm{dt}=\int_{0}^{\infty} \mathrm{t}^{\mathrm{k}+1-1} \mathrm{e}^{-\mathrm{t}} \mathrm{dt}=\Gamma(\mathrm{k}+1)
\end{aligned}
$$

Thus we obtain the $n^{\text {th }}$ ordinary moment of $\mathrm{X}$ for the WeibullLindley distribution as follows:

$$
\mu_{\mathrm{n}}^{\prime}=\eta_{\mathrm{i}, \mathrm{j}, \mathrm{k}, \mathrm{l}, \mathrm{m}, \mathrm{p}}\left[\frac{\Gamma(\mathrm{n}+\mathrm{p}+1)}{(\theta(1+\mathrm{m}))^{\mathrm{n}+\mathrm{p}+1}}+\frac{\Gamma(\mathrm{n}+\mathrm{p}+2)}{(\theta(1+\mathrm{m}))^{\mathrm{n}+\mathrm{p}+2}}\right]
$$

\section{The mean}

The mean of the $W L n D$ can be obtained from the $n^{\text {th }}$ moment of the distribution when $n=1$ as follows:

$$
\begin{array}{rc}
\mu_{\mathrm{n}}^{\prime}=\eta_{\mathrm{i}, \mathrm{j}, \mathrm{k}, \mathrm{l}, \mathrm{m}, \mathrm{p}}\left[\frac{\Gamma(\mathrm{n}+\mathrm{p}+1)}{\left.(\theta(1+\mathrm{m}))^{\mathrm{n}+\mathrm{p}+1}+\frac{\Gamma(\mathrm{n}+\mathrm{p}+2)}{(\theta(1+\mathrm{m}))^{\mathrm{n}+\mathrm{p}+2}}\right]}\right. & \operatorname{Var}(\mathrm{X})=\mathrm{E}\left[\mathrm{X}^{2}\right]-\{\mathrm{E}[\mathrm{X}]\}^{2} \\
\operatorname{Var}(\mathrm{X})=\mu_{2}^{\prime}-\left\{\mu_{1}^{\prime}\right\}^{2} \\
\operatorname{Var}(\mathrm{X})=\eta_{\mathrm{i}, \mathrm{j}, \mathrm{k}, \mathrm{l}, \mathrm{m}, \mathrm{p}}\left[\frac{\Gamma(\mathrm{p}+3)}{(\theta(1+\mathrm{m}))^{\mathrm{p}+3}}+\frac{\Gamma(\mathrm{p}+4)}{(\theta(1+\mathrm{m}))^{\mathrm{p}+4}}\right]-\left\{\eta_{\mathrm{i}, \mathrm{j}, \mathrm{k}, \mathrm{l}, \mathrm{m}, \mathrm{p}}\left[\frac{\Gamma(\mathrm{p}+2)}{(\theta(1+\mathrm{m}))^{\mathrm{p}+2}}+\frac{\Gamma(\mathrm{p}+3)}{(\theta(1+\mathrm{m}))^{\mathrm{p}+3}}\right]\right\}^{2}
\end{array}
$$

\section{The Variance}

The $n^{\text {th }}$ central moment or moment about the mean of $X$, say $\mu_{n}$, can be obtained as

$$
\mu_{\mathrm{n}}=\mathrm{E}\left[\mathrm{X}-\mu_{1}^{\prime}\right]^{\mathrm{n}}=\sum_{\mathrm{i}=0}^{\mathrm{n}}(-1)^{\mathrm{i}}\left(\begin{array}{c}
\mathrm{n} \\
\mathrm{i}
\end{array}\right) \mu_{1}^{\prime \mathrm{i}} \mu_{\mathrm{n}-\mathrm{i}}^{\prime}
$$

The variance of $X$ for $W L n D$ is obtained from the $n^{\text {th }}$ central moment when $n=2$, that is, the variance of $\mathrm{X}$ is the $n^{\text {th }}$ central moment of order two $(\mathrm{n}=2)$ and is given as follows:

The coefficients variation, skewness and kurtosis measures can also be calculated from the non-central moments using some wellknown relationships.

\section{Moment generating function}

The $m g f$ of a random variable $X$ can be obtained by

$$
M_{x}(t)=E\left[e^{t x}\right]=\int_{0}^{\infty} e^{t x} f(x) d x
$$

Using power series expansion in equation (2.2.21) and simplifying the integral therefore we have;

$$
\mathrm{M}_{\mathrm{x}}(\mathrm{t})=\sum_{\mathrm{n}=0}^{\infty} \frac{\mathrm{t}^{\mathrm{n}}}{\mathrm{n} !} \mu_{\mathrm{n}}^{\prime}=\sum_{\mathrm{n}=0}^{\infty} \frac{\mathrm{t}^{\mathrm{n}}}{\mathrm{n} !}\left\{\eta_{\mathrm{i}, \mathrm{j}, \mathrm{k}, \mathrm{l}, \mathrm{m}, \mathrm{p}}\left[\frac{\Gamma(\mathrm{n}+\mathrm{p}+1)}{(\theta(1+\mathrm{m}))^{\mathrm{n}+\mathrm{p}+1}}+\frac{\Gamma(\mathrm{n}+\mathrm{p}+2)}{(\theta(1+\mathrm{m}))^{\mathrm{n}+\mathrm{p}+2}}\right]\right\}
$$

where $n$ and $t$ are constants, $t$ is a real number and $\mu_{n}$ denotes the $n^{\text {th }}$ ordinary moment of $X$.

\section{Characteristics function}

The characteristics function of a random variable $X$ is given by;

$$
\varphi_{\mathrm{x}}(\mathrm{t})=\mathrm{E}\left[\mathrm{e}^{\mathrm{itx}}\right]=\mathrm{E}[\cos (\mathrm{tx})+\mathrm{i} \sin (\mathrm{tx})]=\mathrm{E}[\cos (\mathrm{tx})]+\mathrm{E}[\mathrm{i} \sin (\mathrm{tx})]
$$

Simple algebra and power series expansion proves that

$$
\phi_{\mathrm{x}}(\mathrm{t})=\sum_{\mathrm{n}=0}^{\infty} \frac{(-1)^{\mathrm{n}} \mathrm{t}^{2 \mathrm{n}}}{(2 \mathrm{n}) !} \mu_{2 \mathrm{n}}^{\prime}+\mathrm{i} \sum_{\mathrm{n}=0}^{\infty} \frac{(-1)^{\mathrm{n}} \mathrm{t}^{2 \mathrm{n}+1}}{(2 \mathrm{n}+1) !} \mu_{2 \mathrm{n}+1}^{\prime}
$$

Where $i_{2 n}^{\prime}$ and $i_{2 n+1}^{\prime}$ are the moments of $X$ for $n=2 n$ and $n=2 n+1$ respectively and can be obtained from $i_{n}^{\prime}$ in equation (2.2.17)

\section{Some reliability functions}

In this section, we present some reliability functions associated with $W L n D$ including the survival and hazard functions.

\section{The Survival function}

The survival function describes the likelihood that a system or an individual will not fail after a given time. It tells us about the probability of success or survival of a given product or component. Mathematically, the survival function is given by:

$$
S(x)=1-F(x)
$$

Taking $F(x)$ to be the $c d f$ of the Weibull-Lindley distribution, substituting and simplifying (2.3.1) above, we get the survival function of the $W L n D$ as:

$$
\mathrm{s}(\mathrm{x})=1-\mathrm{e}^{-\alpha\left\{-\log \left[1-\left[1+\frac{\theta \mathrm{x}}{\theta+1}\right] \mathrm{e}^{-\theta \mathrm{x}}\right]\right\}^{\beta}}
$$

Below is a plot of the survival function at chosen parameter values in Figure 3. The figure above revealed that the probability of survival for any random variable following a Weibull-Lindley distribution decreases as the values of the random variable increases, that is, as time goes on, probability of life decreases. This implies that the Weibull-Lindley distribution can be used to model random variables whose survival rate decreases as their age grows. 


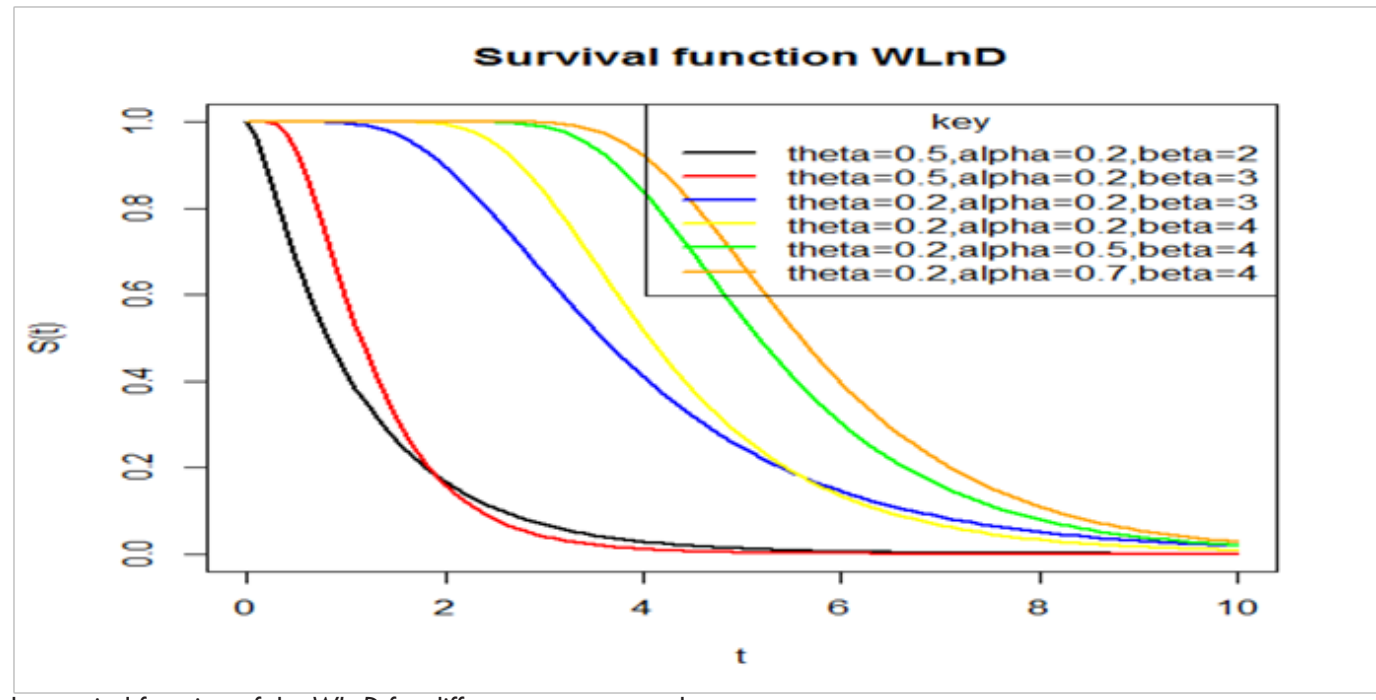

Figure 3 Plot of the survival function of the WLnD for different parameter values.

\section{The Hazard function}

Hazard function as the name implies is also called risk function, it gives us the probability that a component will fail or die for an interval of time. The hazard function is defined mathematically as;

$$
h(x)=\frac{f(x)}{1-F(x)}=\frac{f(x)}{S(x)}
$$

Taking $f(x)$ and $F(x)$ to be the $p d f$ and $c d f$ of the proposed WeibullLindley distribution given previously, we obtain the hazard function as:

$$
\mathrm{h}(\mathrm{x})=\frac{\alpha \beta \theta^{2}(1+\mathrm{x}) \mathrm{e}^{-\theta \mathrm{x}}\left\{-\log \left[1-\left[1+\frac{\theta \mathrm{x}}{\theta+1}\right] \mathrm{e}^{-\theta \mathrm{x}}\right]\right\}^{\beta-1} \mathrm{e}^{-\alpha\left\{-\log \left[1-\left[1+\frac{\theta \mathrm{x}}{\theta+1}\right] \mathrm{e}^{-\theta \mathrm{x}}\right]\right\}^{\beta}}}{(\theta+1)\left[1-\left[1+\frac{\theta \mathrm{x}}{\theta+1}\right] \mathrm{e}^{-\theta \mathrm{x}}\right]\left(1-\mathrm{e}^{\left.-\alpha\left\{\log \left[1-\left[1+\frac{\theta \mathrm{x}}{\theta+1}\right] \mathrm{e}^{-\theta \mathrm{x}}\right]\right]\right\}^{\beta}}\right)}
$$

The following is a plot of the hazard function at chosen parameter values in Figure 4.

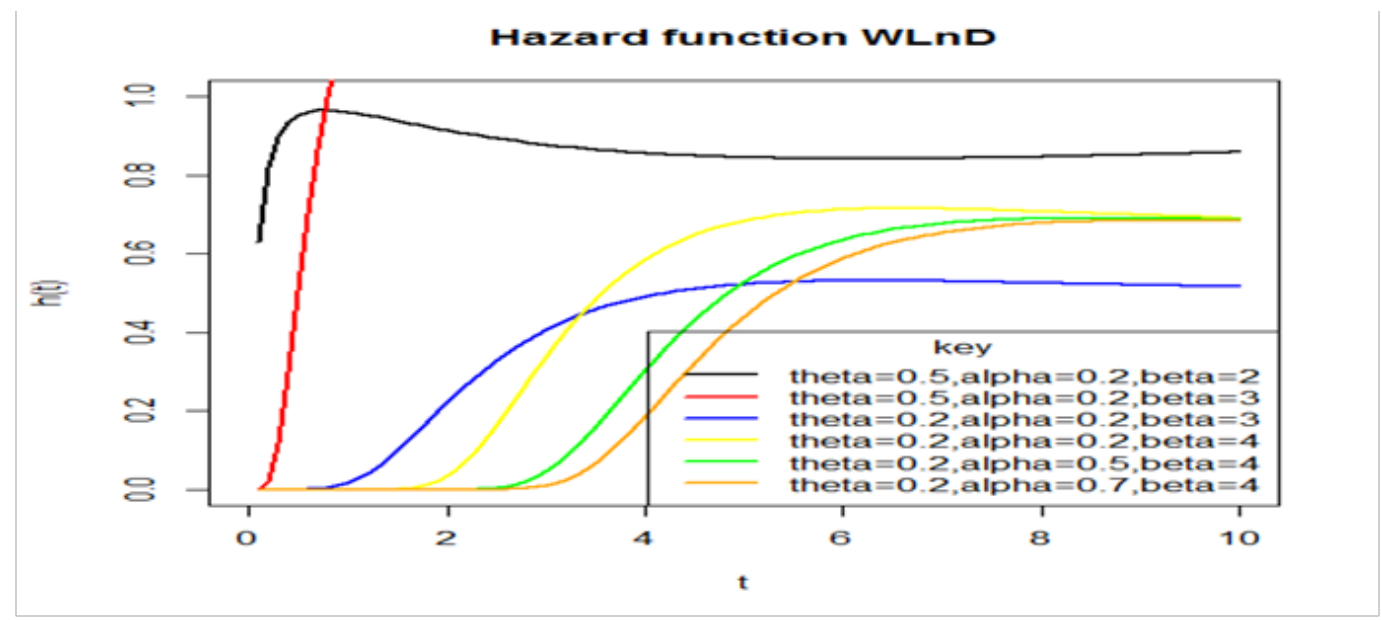

Figure 4 Plot of the hazard function of the $W L n D$ for different parameter values.

Figure 4 above shows the behavior of hazard function of the $W L n D$ and it means that the probability of failure for any Weibull-Lindley random variable increases as the time or age of the variable grows or increases, that is, as time goes, the probability of failure or death increases and becomes constant after some times.

\section{Order statistics}

Order statistics are used widely over the years for solving a huge set of problems such as in robust statistical estimation and detection of outliers, characterization of probability distributions and goodness of fit tests, entropy estimation, analyses of censored samples, reliability analysis, quality control and strength of materials. Suppose $\mathrm{X}_{1}, \mathrm{X}_{2}, \ldots \ldots, \mathrm{X}_{\mathrm{n}}$ is a random sample from a distribution with $p d f$, $f(x)$, and let $\mathrm{X}_{1: \mathrm{n}}<\mathrm{X}_{2: \mathrm{n}}<\ldots \ldots . \mathrm{X}_{\mathrm{a}: \mathrm{n}}$ denote the corresponding order statistic obtained from this sample. The $p d f, \mathrm{f}_{\mathrm{a}: \mathrm{n}}(\mathrm{x})$ of the $\mathrm{a}^{\text {th }}$ order statistic can be defined as;

$$
\mathrm{f}_{\mathrm{a}: \mathrm{n}}(\mathrm{x})=\frac{\mathrm{n} !}{(\mathrm{a}-1) !(\mathrm{n}-\mathrm{a}) !} \sum_{\mathrm{k}=0}^{\mathrm{n}-\mathrm{a}}(-1)^{\mathrm{k}}\left(\begin{array}{c}
\mathrm{n}-\mathrm{a} \\
\mathrm{k}
\end{array}\right) \mathrm{f}(\mathrm{x}) \mathrm{F}(\mathrm{x})^{\mathrm{k}+\mathrm{a}-1}
$$

where $f(x)$ and $F(x)$ are the $p d f$ and $c d f$ of the Weibull-Lindley distribution respectively.

Using (2.1.3) and (2.1.4), the $p d f$ of the $a^{\text {th }}$ order statistics $X_{a: n}$, can be expressed from (2.4.1) as; 


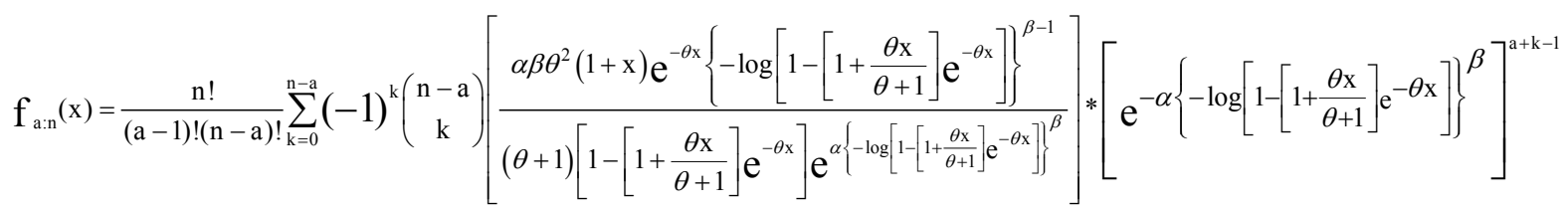

Hence, the $p d f$ of the minimum order statistic $X_{(1)}$ and maximum order statistic $X_{(n)}$ of the $W L n D$ are given by;

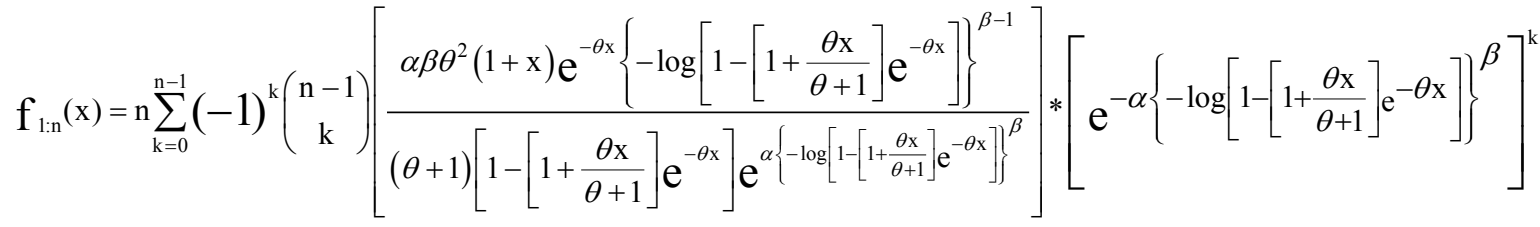

and

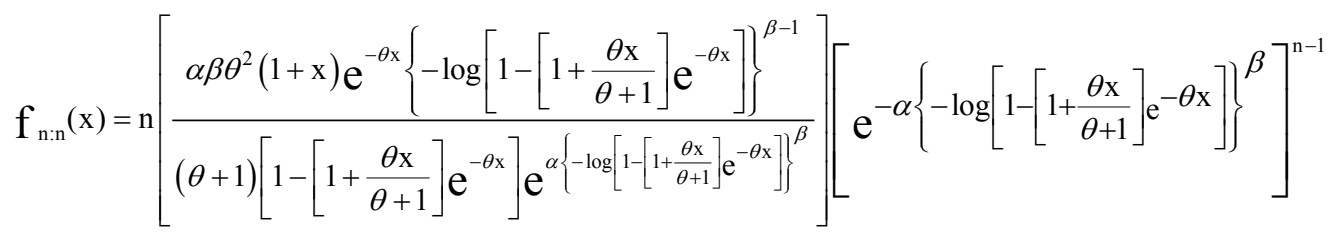

respectively.

\section{Parameter estimation via maximum likelihood}

Let $\mathrm{X}_{1}, \mathrm{X}_{2}, \ldots, \mathrm{X}_{\mathrm{n}}$ be a sample of size ' $\mathrm{n}$ ' independently and

identically distributed random variables from the $W L n D$ with unknown parameters $\alpha, \beta$ and $\theta$ defined previously. The $p d f$ of the $W L n D$ is given from (2.1.3) as

$$
\mathrm{f}(\mathrm{x})=\frac{\alpha \beta \theta^{2}(1+\mathrm{x}) \mathrm{e}^{-\theta \mathrm{x}}\left\{-\log \left[1-\left[1+\frac{\theta \mathrm{x}}{\theta+1}\right] \mathrm{e}^{-\theta \mathrm{x}}\right]\right\}^{\beta-1} \mathrm{e}^{-\alpha\left\{-\log \left[1-\left[1+\frac{\theta \mathrm{x}}{\theta+1}\right] \mathrm{e}^{-\theta \mathrm{x}}\right]\right\}^{\beta}}}{(\theta+1)\left[1-\left[1+\frac{\theta \mathrm{x}}{\theta+1}\right] \mathrm{e}^{-\theta \mathrm{x}}\right]}
$$

The likelihood function is given by;

$$
\mathrm{L}(\underline{\mathrm{X}} \mid \alpha, \beta, \theta)=\frac{\left(\alpha \beta^{\alpha} \theta^{2}\right)^{\mathrm{n}} \prod_{\mathrm{i}=1}^{\mathrm{n}}\left\{\left(1+\mathrm{x}_{\mathrm{i}}\right) \mathrm{e}^{-\theta \mathrm{x}_{\mathrm{i}}}\right\} \prod_{\mathrm{i}=1}^{\mathrm{n}}\left\{-\log \left[1-\left[1+\frac{\theta \mathrm{x}_{\mathrm{i}}}{\theta+1}\right] \mathrm{e}^{-\theta \mathrm{x}_{\mathrm{i}}}\right]\right\}^{\beta-1}}{\left.\left.(\theta+1)^{\mathrm{n}} \prod_{\mathrm{i}=1}^{\mathrm{n}}\left\{1-\left[1+\frac{\theta \mathrm{x}_{\mathrm{i}}}{\theta+1}\right] \mathrm{e}^{-\theta \mathrm{x}_{\mathrm{i}}}\right\} \mathrm{e}^{\alpha \sum_{\mathrm{i}=1}^{\mathrm{n}}\left\{-\log \left[1-\left[1+\frac{\theta \mathrm{x}_{\mathrm{i}}}{\theta+1}\right]\right.\right.} \mathrm{e}^{-\theta \mathrm{x}_{\mathrm{i}}}\right]\right\}^{\beta}}
$$

Let the $\log$-likelihood function, $1=\log \mathrm{L}(\underline{\mathrm{X}} \mid \alpha, \beta, \theta)$ therefore

$$
\begin{aligned}
& 1=\mathrm{n} \log \alpha+\mathrm{n} \log \beta+2 \mathrm{n} \log \theta-\mathrm{n} \log (\theta+1)+\sum_{\mathrm{i}=1}^{\mathrm{n}} \log \left(1+\mathrm{x}_{\mathrm{i}}\right)-\theta \sum_{\mathrm{i}=1}^{\mathrm{n}} \mathrm{x}_{\mathrm{i}}+(\beta-1) \sum_{\mathrm{i}=1}^{\mathrm{n}} \log \left\{-\log \left[1-\left[1+\frac{\theta \mathrm{x}_{\mathrm{i}}}{\theta+1}\right] \mathrm{e}^{-\theta \mathrm{x}_{\mathrm{i}}}\right]\right\} \\
& -\sum_{\mathrm{i}=1}^{\mathrm{n}} \log \left(\left[1-\left[1+\frac{\theta \mathrm{x}_{\mathrm{i}}}{\theta+1}\right] \mathrm{e}^{-\theta \mathrm{x}_{\mathrm{i}}}\right]\right)-\alpha \sum_{\mathrm{i}=1}^{\mathrm{n}}\left\{-\log \left[1-\left[1+\frac{\theta \mathrm{x}_{\mathrm{i}}}{\theta+1}\right] \mathrm{e}^{-\theta \mathrm{x}_{\mathrm{i}}}\right]\right\}^{\beta}
\end{aligned}
$$

Differentiating $\boldsymbol{l}$ partially with respect to $\alpha, \beta$ and $\theta$ respectively gives;

$$
\begin{aligned}
& \frac{\partial \mathrm{l}}{\partial \alpha}=\frac{\mathrm{n}}{\alpha}-\sum_{\mathrm{i}=1}^{\mathrm{n}}\left\{-\log \left[1-\left[1+\frac{\theta \mathrm{x}_{\mathrm{i}}}{\theta+1}\right] \mathrm{e}^{-\theta \mathrm{x}_{\mathrm{i}}}\right]\right\}^{\beta} \\
& \frac{\partial \mathrm{l}}{\partial \beta}=\frac{\mathrm{n}}{\beta}+\sum_{\mathrm{i}=1}^{\mathrm{n}} \log \left\{-\log \left[1-\left[1+\frac{\theta \mathrm{x}_{\mathrm{i}}}{\theta+1}\right] \mathrm{e}^{-\theta \mathrm{x}_{\mathrm{i}}}\right]\right\}-\alpha \sum_{\mathrm{i}=1}^{\mathrm{n}}\left\{-\log \left[1-\left[1+\frac{\theta \mathrm{x}_{\mathrm{i}}}{\theta+1}\right] \mathrm{e}^{-\theta \mathrm{x}_{\mathrm{i}}}\right]\right\}^{\beta}\left\{-\log \left[1-\left[1+\frac{\theta \mathrm{x}_{\mathrm{i}}}{\theta+1}\right] \mathrm{e}^{-\theta \mathrm{x}_{\mathrm{i}}}\right]\right\}
\end{aligned}
$$




$$
\begin{aligned}
& \frac{\partial \mathrm{l}}{\partial \theta}=\frac{2 \mathrm{n}}{\theta}-\frac{\mathrm{n}}{\theta+1}-\sum_{\mathrm{i}=1}^{\mathrm{n}} \mathrm{x}_{\mathrm{i}}+(\beta-1) \sum_{\mathrm{i}=1}^{\mathrm{n}}\left\{\frac{\mathrm{x}_{\mathrm{i}} \mathrm{e}^{-\theta \mathrm{x}_{\mathrm{i}}}\left[\frac{1}{(\theta+1)^{2}}-\frac{\theta \mathrm{x}_{\mathrm{i}}+\theta+1}{\theta+1}\right]}{\left\{-\log \left[1-\left[1+\frac{\theta \mathrm{x}_{\mathrm{i}}}{\theta+1}\right] \mathrm{e}^{-\theta \mathrm{x}_{\mathrm{i}}}\right]\right\}\left\{\left[1-\left[1+\frac{\theta \mathrm{x}_{\mathrm{i}}}{\theta+1}\right] \mathrm{e}^{-\theta \mathrm{x}_{\mathrm{i}}}\right]\right\}}\right\}-\sum_{\mathrm{i}=1}^{\mathrm{n}}\left\{\frac{\mathrm{x}_{\mathrm{i}} \mathrm{e}^{-\theta \mathrm{x}_{\mathrm{i}}}\left[\frac{1}{(\theta+1)^{2}}-\frac{\theta \mathrm{x}_{\mathrm{i}}+\theta+1}{\theta+1}\right]}{\left(\left[1-\left[1+\frac{\theta \mathrm{x}_{\mathrm{i}}}{\theta+1}\right] \mathrm{e}^{-\theta \mathrm{x}_{\mathrm{i}}}\right]\right)}\right\} \\
& +\alpha \beta \sum_{\mathrm{i}=1}^{\mathrm{n}}\left\{\frac{\mathrm{x}_{\mathrm{i}} \mathrm{e}^{-\theta \mathrm{x}_{\mathrm{i}}\left[\frac{1}{(\theta+1)^{2}}-\frac{\theta \mathrm{x}_{\mathrm{i}}+\theta+1}{\theta+1}\right]\left\{-\log \left[1-\left[1+\frac{\theta \mathrm{x}_{\mathrm{i}}}{\theta+1}\right] \mathrm{e}^{-\theta \mathrm{x}_{\mathrm{i}}}\right]\right\}^{\beta-1}}}{\left[1-\left[1+\frac{\theta \mathrm{x}_{\mathrm{i}}}{\theta+1}\right] \mathrm{e}^{-\theta \mathrm{x}_{\mathrm{i}}}\right]}\right\}
\end{aligned}
$$

Equating equations (2.5.3), (2.5.4) and (2.5.5) to zero and solving for the solution of the non-linear system of equations will give us the maximum likelihood estimates of parameters $\alpha, \beta \& \theta$ respectively. However, the above equations cannot be solved manually due to their complexity unless numerically with the help of statistically inclined computer programs like Python, R, SAS, etc., when data sets are available.

\section{Results and discussions}

This section presents four datasets, their descriptive statistics, graphics and applications to some selected extensions of the Lindley

Table I Summary statistics for dataset I distribution including the classical Lindley distribution. We have compared the performance of the Weibull-Lindley distribution $(W L l D)$ to some families of Lindley distribution such as LomaxLindley distribution ( $L L I D)$, Two-parameter Lindley distribution (TPLID), Transmuted Lindley distribution (TLID) and the Lindley distribution $(L l D)$.

\section{Data sets and their nature}

In this section, four different datasets and their summary are presented for fitting the above listed distributions. The available data sets I, II, III, and IV and their respective summary statistics are provided in Table $1-5$ respectively as follows;

\begin{tabular}{lllllllllll}
\hline Parameters & $\boldsymbol{n}$ & Minimum & $\mathrm{Q}_{1}$ & Median & $\mathrm{Q}_{3}$ & Mean & Maximum & Variance & Skewness & Kurtosis \\
\hline Values & 20 & 1.1 & 1.475 & 1.7 & 2.05 & 1.9 & 4.1 & 0.4958 & 1.8625 & 7.1854 \\
\hline
\end{tabular}

Table 2 Summary statistics for dataset II

\begin{tabular}{lllllllllll}
\hline Parameters & $\boldsymbol{n}$ & Minimum & $\mathrm{Q}_{1}$ & Median & $\mathrm{Q}_{3}$ & Mean & Maximum & Variance & Skewness & Kurtosis \\
\hline Values & 20 & 40 & 86.75 & 119 & 140.8 & 113.45 & 165 & 1280.892 & -0.3552 & -0.89 \\
\hline
\end{tabular}

Table 3 Summary Statistics for data set III

\begin{tabular}{lllllllllll}
\hline Parameters & $\mathbf{n}$ & Minimum & $\mathrm{Q}_{1}$ & Median & $\mathrm{Q}_{3}$ & Mean & Maximum & Variance & Skewness & Kurtosis \\
\hline Data set I & 63 & 0.55 & 1.375 & 1.59 & 1.685 & 1.507 & 2.24 & 0.105 & -0.8786 & 3.9238 \\
\hline
\end{tabular}

Table 4 Descriptive statistics for dataset IV

\begin{tabular}{lllllllllll}
\hline Parameters & $\boldsymbol{n}$ & Minimum & $\mathrm{Q}_{1}$ & Median & $\mathrm{Q}_{3}$ & Mean & Maximum & Variance & Skewness & Kurtosis \\
\hline Values & 59 & 4.1 & 8.45 & 10.6 & 16.85 & 13.49 & 39.2 & 64.8266 & 1.6083 & 2.256 \\
\hline
\end{tabular}

Dataset I: This dataset represents the lifetime's data relating to relief times (in minutes) of 20 patients receiving an analgesic and reported by Gross et al., ${ }^{34}$ and has been used by Shanker et al., ${ }^{35}$ Table 1 .

Dataset II: This data represent the survival times in weeks for male rats Lawless et al., ${ }^{36}$ (Table 2).

Data set III: This data set represents the strength of $1.5 \mathrm{~cm}$ glass fibers initially collected by members of staff at the UK national laboratory. It has been used by Bourguignon et al., ${ }^{18}$ Afify et al., ${ }^{37}$ Barreto Souza et al. ${ }^{38}$ Oguntunde et al., ${ }^{39}$ Ieren et al., ${ }^{40}$ as well as Smith et al., ${ }^{41}$ Its summary is given as follows: (Table 3).

Dataset IV: This dataset represents 59 observations of the monthly actual taxes revenue in Egypt (in 1,000 million Egyptian pounds) between January 2006 and November 2010. The data has been previously used by Owoloko et al..$^{42}$ The descriptive statistics for this data are as follows:

We also provide some histograms and densities for the three data sets as shown in Figure 5-8 below respectively (Table 4). 

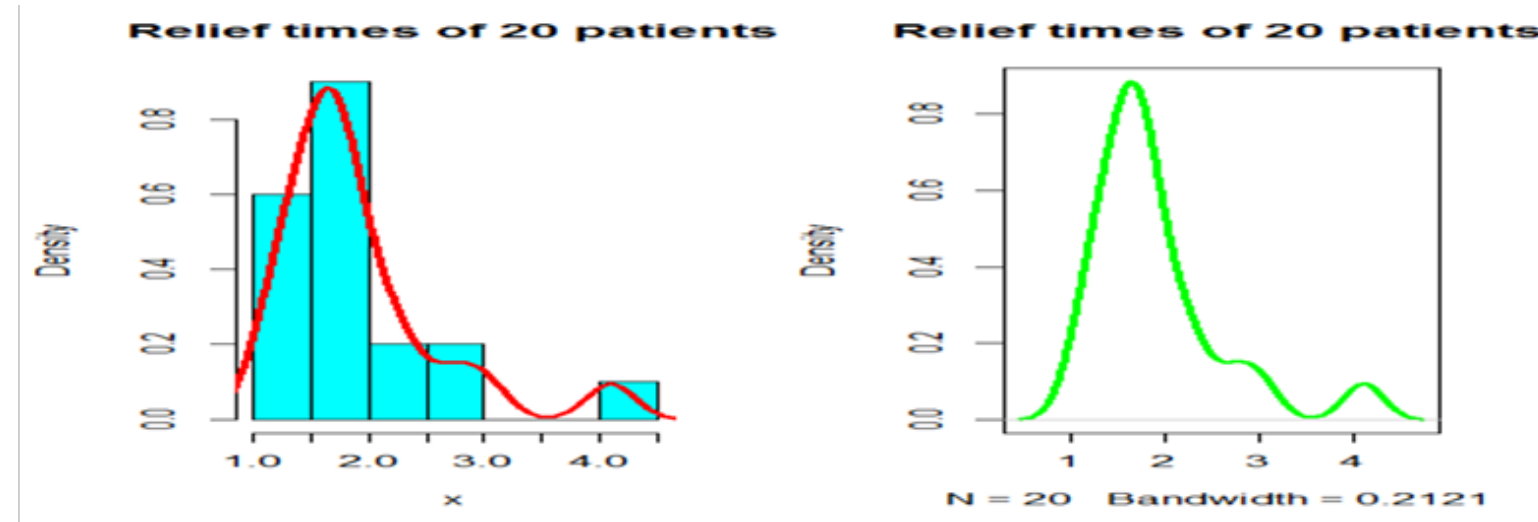

Figure 5 Histogram and density plot for the Relief times of 20 patients (Data set I).
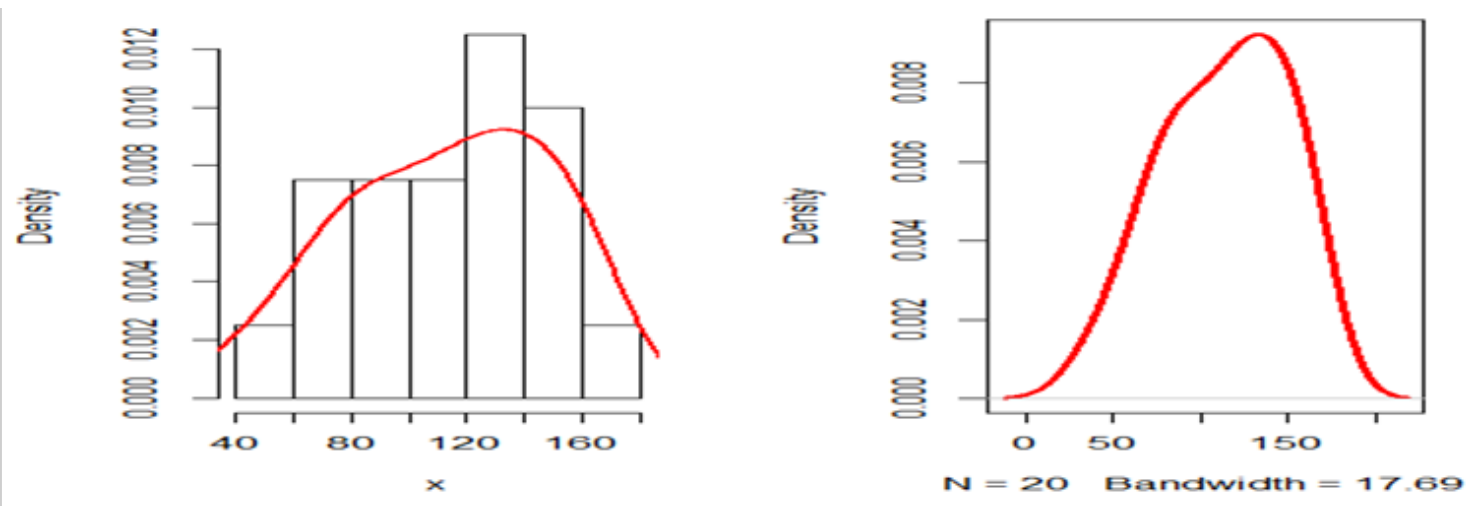

Figure 6 Histogram and density plot for the survival times in weeks for male rats (Data set II).
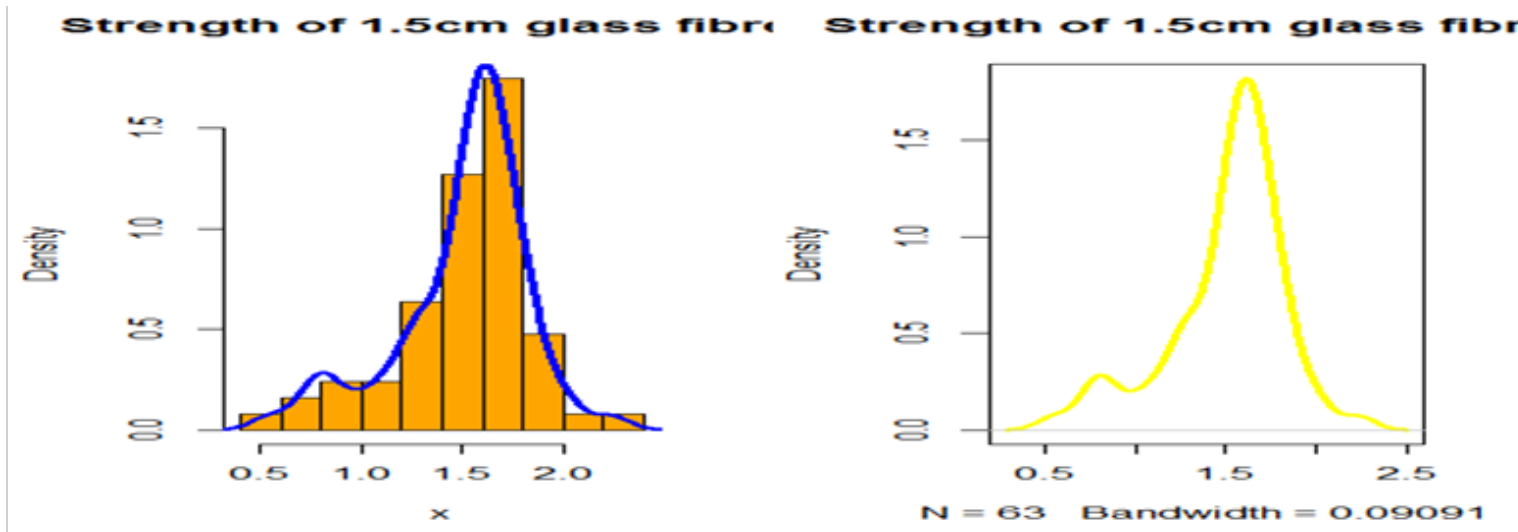

Figure $7 \mathrm{~A}$ histogram and density plot for the strength of $1.5 \mathrm{~cm}$ glass fibres (Data set III).
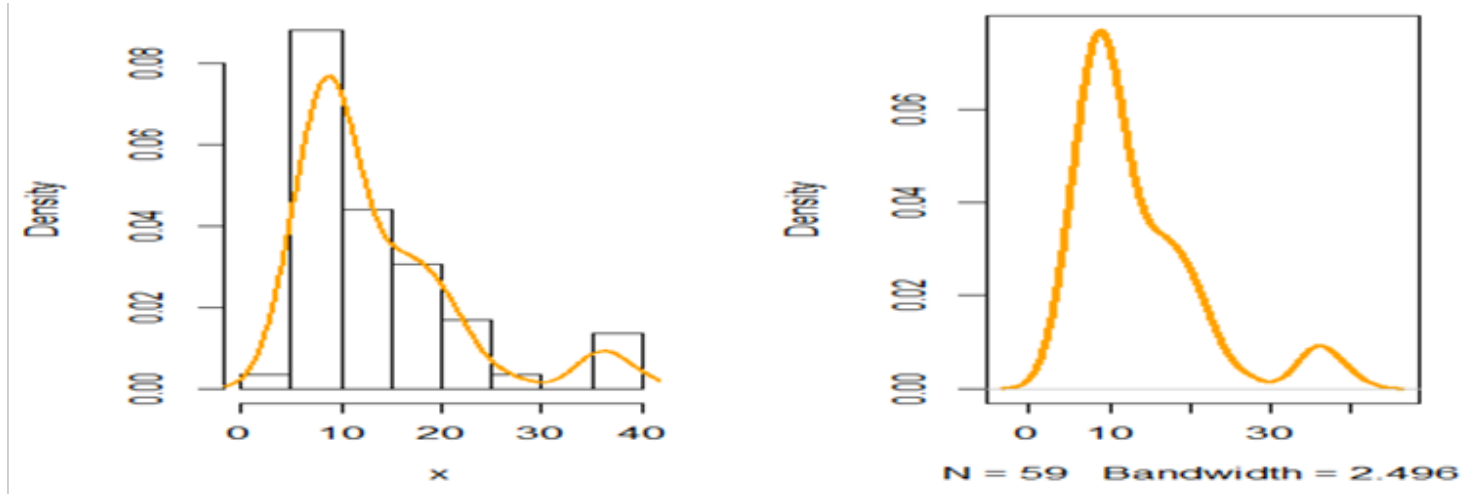

Figure $8 \mathrm{~A}$ histogram and density plot for the monthly actual taxes revenue in Egypt (Data set IV).

Citation: Ieren TG, Oyamakin SO, Chukwu AU. Modeling lifetime data with Weibull-Lindley distribution. Biom Biostat Int J. 20I8;7(6):532-544. DOI: I0.15406/bbij.2018.07.00256 
From the summary statistics of the four data sets, we found that data sets I and IV are positively skewed, while II is a bit negatively skewed or approximately normal and III is negatively skewed. Also, data sets I, III and IV have higher kurtosis while II have low level or degree of peakness.

\section{Analysis of data}

These four different datasets presented above were used to fit all the above listed Lindley distributions by applying the formulas of the test statistics in section 2 in order to get the best fitted model and the results are presented as follow in the four tables for each dataset below: (Table 5).

From Table 5, the values of the parameter MLEs and the corresponding values of $l l, A I C, A^{*}, W^{*}$ and $K-S$ for each model show that the Weibull-Lindley distribution (WLID) has better performance compared to the other four models namely: Lomax-Lindley distribution (LLID), Two-parameter Lindley distribution (TPLID), Transmuted Lindley distribution (TLID) and the Lindley distribution $(L I D)$ and hence becomes the best fitted distribution based the data set I (Table 6).

Table 5 The statistics II, AIC, $A^{*}, W^{*}$ and $K$-S for the fitted models to the first dataset

\begin{tabular}{|c|c|c|c|c|c|c|c|}
\hline \multirow{2}{*}{ Parameter estimates } & \multirow{2}{*}{ ll=(log-likelihood value) } & \multirow{2}{*}{ AIC } & \multirow{2}{*}{$A^{*}$} & \multirow{2}{*}{$W^{*}$} & \multirow{2}{*}{ K-S } & P-Value & \multirow{2}{*}{ Ranks } \\
\hline & & & & & & $(K-S)$ & \\
\hline \multicolumn{8}{|l|}{0.5842} \\
\hline 1.2337 & 16.0004 & 38.0009 & 0.2483 & 0.0428 & 0.167 & 0.6324 & I \\
\hline \multicolumn{8}{|l|}{3.8112} \\
\hline \multicolumn{8}{|l|}{ I.I589 } \\
\hline-0.9888 & 24.9726 & 53.9452 & 0.6295 & 0.1063 & 0.3113 & 0.0414 & 2 \\
\hline \multicolumn{8}{|l|}{0.8926} \\
\hline 9.7008 & 27.2805 & 58.561 & 0.6401 & 0.108 & 0.2885 & 0.0716 & 3 \\
\hline 0.8162 & 30.2496 & 62.4991 & 0.6758 & 0.1141 & 0.3911 & 0.0044 & 4 \\
\hline \multicolumn{8}{|l|}{0.361} \\
\hline 9.6021 & 29.8421 & 65.6841 & 0.6552 & 0.1107 & 0.416 & 0.002 & 5 \\
\hline 2.4483 & & & & & & & \\
\hline
\end{tabular}

Table 6 The statistics II, AIC, $A^{*}, W^{*}$ and $K$-S for the fitted models to the second dataset

\begin{tabular}{|c|c|c|c|c|c|c|c|}
\hline \multirow{2}{*}{$\begin{array}{l}\text { Parameter } \\
\text { estimates }\end{array}$} & \multirow{2}{*}{ Il=(log-likelihood value $)$} & \multirow{2}{*}{$A I C$} & \multirow{2}{*}{$A^{*}$} & \multirow{2}{*}{$W^{*}$} & \multirow{2}{*}{$K-S$} & P-Value & \multirow{2}{*}{ Ranks } \\
\hline & & & & & & $(K-S)$ & \\
\hline \multicolumn{8}{|l|}{0.0278} \\
\hline 1.6862 & 106.6467 & 219.2935 & 0.4311 & 0.062 & 0.3225 & 0.0313 & I \\
\hline \multicolumn{8}{|l|}{1.0152} \\
\hline \multicolumn{8}{|l|}{0.0198} \\
\hline 1.9445 & || $2.889 \mid$ & 231.7781 & 0.5199 & 0.076 & 0.3772 & 0.0068 & 2 \\
\hline \multicolumn{8}{|l|}{2.8037} \\
\hline 0.0371 & & & & & & & \\
\hline 0.3054 & 128.3464 & 260.6929 & 0.4103 & 0.0586 & $0.694 I$ & 8.57E-09 & 3 \\
\hline 1.9987 & & & & & & & \\
\hline 0.6326 & 4442.078 & 8888.156 & $\mathrm{NaN}$ & $\mathrm{NaN}$ & 1 & $<2.2 \mathrm{e}-16$ & 5 \\
\hline 2.2161 & 4926.226 & 9854.452 & $\mathrm{NaN}$ & $\mathrm{NaN}$ & 1 & $<2.2 \mathrm{e}-16$ & 4 \\
\hline
\end{tabular}

Again the results in Table 6 above shows that the Weibull-Lindley distribution $(W L I D)$ fits the second dataset better than the other four models (LLID, TPLID, TLID and LID) because the values of the statistics; $A I C, A^{*}, W^{*}$ and $K-S$ are smaller for the WLID than the other models and therefore it is considered as the best fitted distribution based the data set II (Table 7).
The results from Table 7 also agrees with the previous results that the WLID is more flexible compared to the three other models this also agrees with the fact that generalizing any continuous distribution provides a compound distribution with at least better fit than the classical distribution (i.e Lindley) irrespective of the nature of the data used provide it is asymmetry Table 8 . 
Table 7 The statistics II, AIC, $A^{*}, W^{*}$ and $K-S$ for the fitted models to the third dataset

\begin{tabular}{|c|c|c|c|c|c|c|c|c|}
\hline \multirow{2}{*}{ Distributions } & \multirow{2}{*}{$\begin{array}{l}\text { Parameter } \\
\text { estimates }\end{array}$} & \multirow{2}{*}{ ll=(log-likelihood value) } & \multirow{2}{*}{$A I C$} & \multirow{2}{*}{$A^{*}$} & \multirow{2}{*}{$\mathbf{W}^{*}$} & \multirow{2}{*}{$K-S$} & & \multirow{2}{*}{ Ranks } \\
\hline & & & & & & & $(K-S)$ & \\
\hline & 1.4523 & & & & & & & \\
\hline & 7.6251 & 34.1708 & 74.3416 & 4.2254 & 0.7768 & 0.224 & 0.0033 & I \\
\hline & $\mathrm{I} .7248$ & & & & & & & \\
\hline & $0.36 I$ & & & & & & & \\
\hline & 9.6021 & $81.47 \mid 4$ & 168.9428 & 3.074 & 0.5619 & 0.3213 & $3.60 \mathrm{E}-06$ & 2 \\
\hline & 2.4483 & & & & & & & \\
\hline & 1.391 & & & & & & & \\
\hline & -0.9937 & 63.8482 & 131.6963 & 3.1901 & 0.5833 & 0.3413 & 6.70E-07 & 3 \\
\hline & 1.2155 & & & & & & & \\
\hline & 9.2573 & 71.0355 & |46.07| & $3.14 \mid 4$ & 0.5744 & 0.3427 & $5.90 \mathrm{E}-07$ & 4 \\
\hline & 0.9957 & 82.5853 & 167.1706 & 3.0788 & 0.563 & 0.3885 & 8.20E-09 & 5 \\
\hline
\end{tabular}

Table 8 The statistics II, AIC, $A^{*}, W^{*}$ and K-S for the fitted models to the fourth dataset

\begin{tabular}{|c|c|c|c|c|c|c|c|c|}
\hline Distributions & $\begin{array}{l}\text { Parameter } \\
\text { estimates }\end{array}$ & ll=(log-likelihood value) & AIC & $A^{*}$ & $W^{*}$ & $K-S$ & $\begin{array}{l}\text { P-Value } \\
\text { (K-S) }\end{array}$ & Ranks \\
\hline & 0.3767 & \multirow{4}{*}{199.8163} & \multirow{3}{*}{405.6327} & \multirow{3}{*}{0.7927} & \multirow{3}{*}{0.1329} & \multirow{3}{*}{0.1597} & \multirow{3}{*}{0.0986} & \multirow{3}{*}{ I } \\
\hline & 8.5414 & & & & & & & \\
\hline & 0.8256 & & & & & & & \\
\hline & 0.0809 & & \multirow{3}{*}{408.1067} & \multirow{3}{*}{ I. 127} & \multirow{3}{*}{0.1849} & \multirow{3}{*}{0.1558} & \multirow{3}{*}{0.1139} & \multirow{3}{*}{2} \\
\hline & 6.6768 & \multirow[t]{2}{*}{201.0534} & & & & & & \\
\hline & 3.1649 & & & & & & & \\
\hline & $\begin{array}{l}0.1429 \\
-0.4154\end{array}$ & 199.6626 & $403.325 I$ & $\mathrm{I} .425 \mathrm{I}$ & 0.229 & 0.1676 & 0.0728 & 5 \\
\hline & 0.1618 & & & & & & & \\
\hline & 4.938 & 199.324 & 402.648 & 1.2489 & 0.2007 & 0.2084 & 0.0119 & 3 \\
\hline & 0.1361 & 200.6599 & 403.3198 & 1.2999 & 0.2087 & 0.1844 & 0.0361 & 4 \\
\hline
\end{tabular}

Lastly, our results in Table 8 provides the same results as obtained in the above previous tables with the Weibull-Lindley distribution performing better than the other three distributions considered in this study.

The following figures displayed the histogram and estimated densities of the fitted models for the four real life data sets used in this study.

From the estimated density plots in Figures 9 we can observe that though there is no big difference between the performance of the other four models, it is very clear that the performance of the Weibull-Lindley distribution (WLID) remains the best and consistent irrespective of the nature the datasets as compared to the LomaxLindley distribution (LLID), Two-parameter Lindley distribution (TPLID), Transmuted Lindley distribution (TLID) and the Lindley distribution $(L l D)$.

Furthermore, the performance of the Weibull-Lindley could be attributed to the fact that the Weibull-Lindley distribution is heavytailed and highly skewed to the right with excellent flexibility which allows it to take various shapes depending on the parameter values and it also exhibit some degree of kurtosis all of which are features of the four datasets used in this research, hence, the Weibull-Lindley distribution will be more appropriate for lifetime datasets which are positively skewed with a higher degree of peakness as well as those that are approximately normal with observations above zero.

Hence, having demonstrated earlier in Tables 5-8, we have a similar conclusion based on figure 3.5 that the Weibull-Lindley distribution has a better fit for the four data sets considered in this study. 

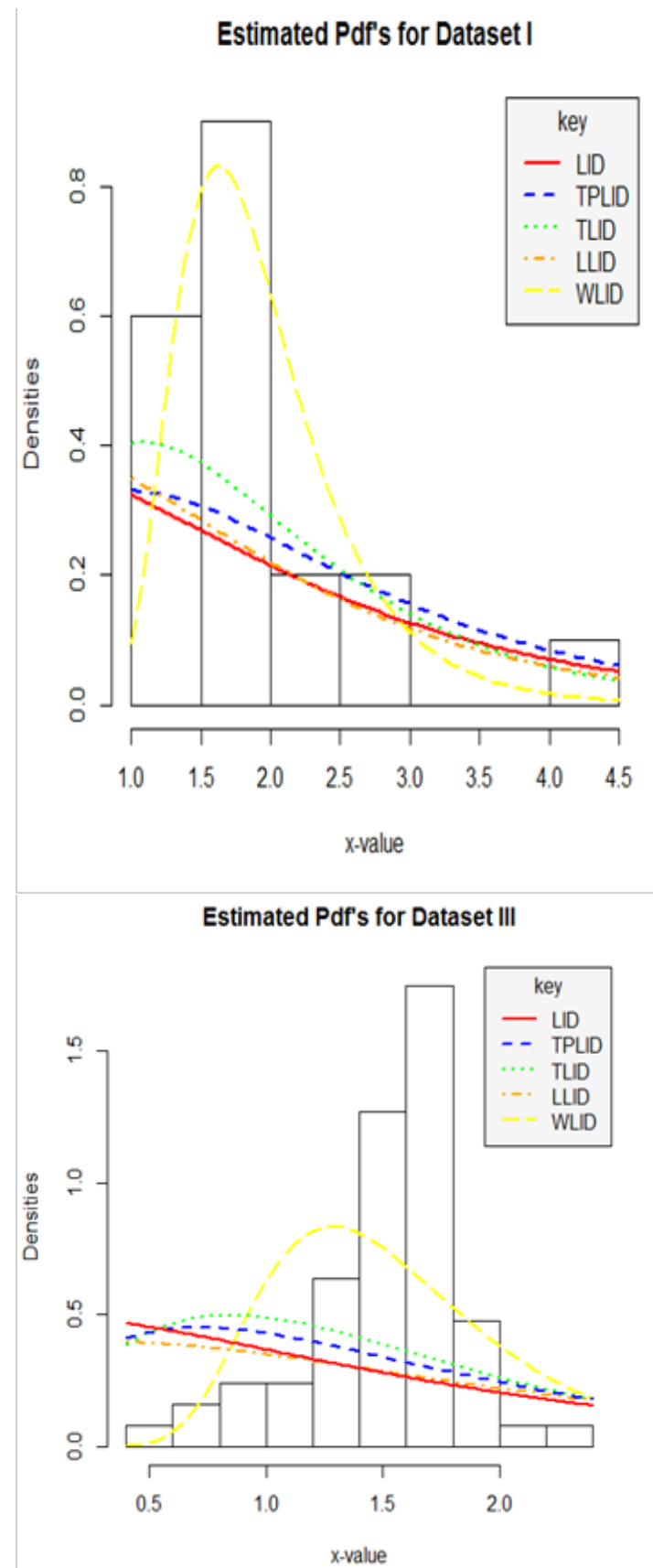

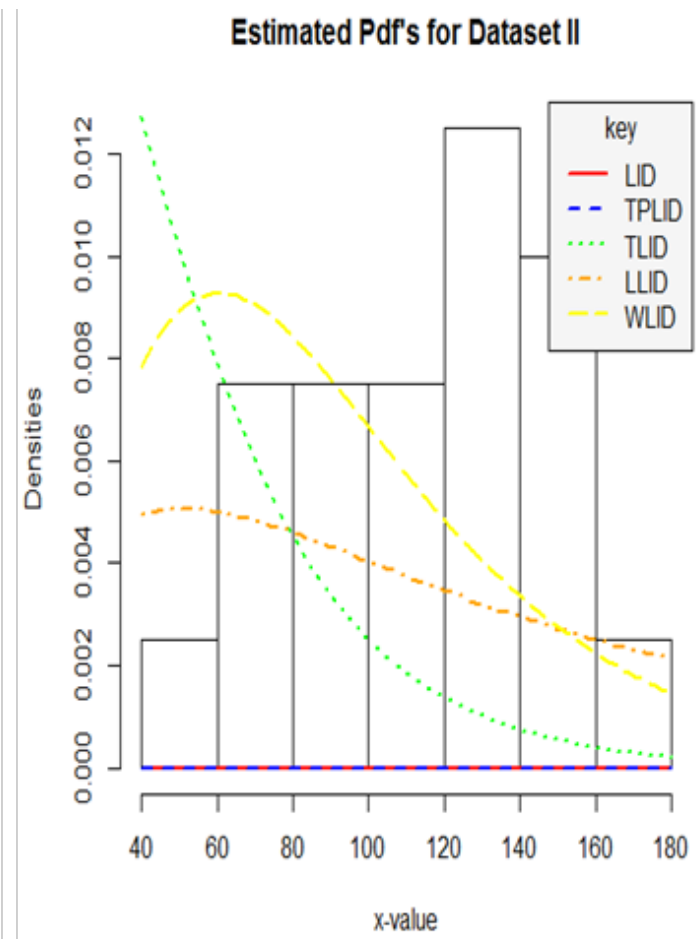

Estimated Pdf's for Dataset IV

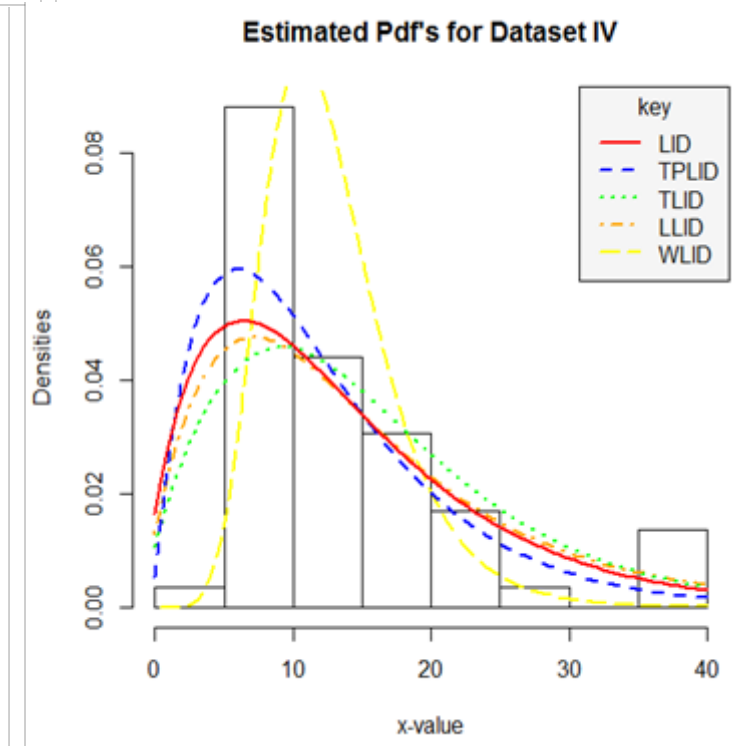

Figure 9 Histogram and estimated densities of the Weibull-Lindley distribution (WLID), Lomax-Lindley distribution (LLID), Two-parameter Lindley distribution (TPLID), Transmuted Lindley distribution (TLID) and the Lindley distribution (LID) for the four real life datasets (dataset I, II, III and IV).

\section{Summary conclusion}

This article proposed a new distribution called Weibull-Lindley distribution. The Mathematical and Statistical properties of the new distribution have been derived and studied extensively. Some of its properties with graphical analysis and discussion on their usefulness and applications were also considered. The model parameters were estimated using maximum likelihood method and we have a conclusion based on our applications of the model to four real life datasets that the new distribution $(W L n D)$ has a better fit compared to the other four already existing models considered in this study.

\section{Acknowledgments}

None.

\section{Conflicts of interest}

Author declares that there is no conflicts of interest.

\section{References}

1. Tahir MH, Zubair M, Mansoor M, et al. A new Weibull-G family of distributions. Hacettepe Journal of Mathematics and Statistics. 2016;45(2):629-647.

2. Lindley DV. Fiducial distributions and Bayes' theorem. Journal Royal Statistical Society Series B. 1958;20:102-107.

3. Ghitany ME, Atieh B, Nadarajah S. Lindley distribution and its applications. Mathematical Computation and Simulation. 2008;78(4):493-506. 
4. Eugene N, Lee C, Famoye F. Beta-Normal Distribution and It Applications. Communication in Statistics: Theory and Methods. 2002;31:497-512.

5. Cordeiro GM, De Castro M. A New Family of Generalized Distributions. Journal Statistical Computation and Simulation. 2001;81:883-898.

6. Shaw WT, Buckley IR. The alchemy of probability distributions: beyond Gram-Charlier expansions and a skew-kurtotic-normal distribution from a rank transmutation map. Research report. 2007.

7. Zografos K, Balakrishnan N. On families of beta-and generalized gamma generated distributions and associated inference. Statistical Methods. 2009;63:344-362.

8. Alexander C, Cordeiro GM, Ortega EMM, et al. Generalized betagenerated distributions. Computational Statistics and Data Analysis. 2012;56:1880-1897.

9. Ristic MM, Balakrishnan N. The gamma-exponentiated exponential distribution. Journal Statistics Computation and Simulation. 2012;82:1191-1206.

10. Torabi H, Montazari NH. The gamma-uniform distribution and its application. Kybernetika. 2012;48:16-30

11. Amini M, Mirmostafaee SMTK, Ahmadi J. Log-gamma-generated families of distributions. Statistics. 2014;48(4):913-932.

12. Alzaghal A, Lee C, Famoye F. Exponentiated T-X family of distributions with some applications. International Journal Probability and Statistics. 2013;2:31-49.

13. Cordeiro GM, Ortega EMM, Da Cunha DCC. The exponentiated generalized class of distribution. Journal of Data Science. 2013;11:1-27.

14. Torabi H, Montazari NH. The logistic-uniform distribution and its application. Communications Statistics: Simulation and Computation. 2014;43:2551-2569.

15. Alzaatreh A, Famoye F, Lee C. The gamma-normal distribution: Properties and applications. Computational Statistics and Data Analysis. 2014;69:67-80.

16. Tahir MH, Cordeiro GM, Alzaatreh A, et al. The logistic-X family of distributions and its applications. Communication in Statistics: Theory and Methods. 2016;45(24):7326-7349.

17. Alzaatreh A, Famoye F, Lee C. Weibull-Pareto distribution and its applications. Communication in Statistics: Theory and Methods. 2013;42:1673-1691.

18. Bourguignon M, Silva RB, Cordeiro GM. The Weibull-G Family of Probability distributions. Journal Data Science. 2014;12: 53-68.

19. Cordeiro GM, Ortega EMM, Popovic BV, et al. The Lomax generator of distributions: Properties, minification process and regression model. Applied Mathematics and Computation. 2014;247:465-486.

20. Cordeiro GM, Ortega EMM, Ramires TG. A new generalized Weibull family of distributions: Mathematical properties and applications. Journal Statistical Distributionand Applications. 2015;2(13):13.

21. Alizadeh M, Cordeiro GM, De Brito E, et al. The beta Marshall-Olkin family of distributions. Journal of Statistical Distribution Application. 2015;2(4):1-18.

22. Merovci C. Transmuted Lindley distribution. International Journal of Open Problems in Computational Mathematics. 2003;6(2):63-74.

23. Ashour SK, Eltehiwy MA. Exponentiated power Lindley distribution. Journal of Advanced Research. 2005;6:895-905.
24. Nadarajah S, Bakouch HS, Tahmasbi R. A generalized Lindley distribution. Sankhya B. 2015;73:331-359.

25. Elgarhy M, M.Rashed M, Shawki AW. Transmuted Generalized Lindley Distribution. International Journal of Mathematics Trends and Technology. 2016;29(2):145-154.

26. Alkarni SH. Extended Power Lindley Distribution: A New Statistical Model For Non-Monotone Survival Data. European Journal of Statistics and Probability. 2015;3(3):19-34.

27. Shanker R, Kamlesh KK, Fesshaye H. A Two Parameter Lindley Distribution: Its Properties and Applications. Biostatistics and Biometrics Open Access Journal. 2017;1(4):555570.

28. Yahaya A, Lawal A. A study on Lomax-Lindley distribution with applications to simulated and real life data. Nigeria: University of Calabar, Calabar; 2018.

29. Al-khazaleh M, Al-Omari AI, Al-khazaleh AMH. Transmuted TwoParameter Lindley Distribution. Journal of Statistics Applications and Probability. 2016;5(3):421-432

30. Shanker R, Shukla KK, Shanker R, et al. A Three-Parameter Lindley Distribution. American Journal of Mathematics and Statistics. 2017;7(1):15-26.

31. Hyndman RJ, Fan Y. Sample quantiles in statistical packages. The American Statistics. 1996;50(4):361-365.

32. Kenney JF, Keeping ES. Mathematics of Statistics. $3^{\text {rd }}$ edn. New Jersey: Chapman \& Hall Ltd. 1962.

33. Moors JJ. A quantile alternative for kurtosis. Journal of the Royal Statistical Society. 1988;37:25-32.

34. Gross AJ, Clark VA. Survival distributions reliability applications in the Biometrical Sciences. New York: John Wiley; 1975.

35. Shanker R, Fesshaye H, Sharma S. On two-Parameter Lindley distribution and its applications to model lifetime data. Biom Biostat Int J. 2016;3(1):00056.

36. Lawless JF. Statistical Models and Methods for Lifetime Data. $2^{\text {nd }}$ edn. New Jersey: Wiley; 2003.

37. Afify AZ, Aryal G. The Kummaraswamy exponentiated Frechet distribution. Journal of Data Science. 2016;6:1-19.

38. Barreto Souza WM, Cordeiro GM, Simas AB. Some results for beta Frechet distribution. Communication in Statistics: Theory and Methods. 2011;40:798-811

39. Oguntunde PE, Balogun OS, Okagbue HI, et al. The Weibull-Exponential Distribution: Its Properties and Applications. Journal of Applied Science. 2015;15(11):1305-1311.

40. Ieren TG, Yahaya A. The Weimal Distribution: its properties and applications. Journal of the Nigeria Association of Mathematical Physics. 2017;39:135-148.

41. Smith RL, Naylor JC. A Comparison of Maximum Likelihood and Bayesian Estimators for the Three-Parameter Weibull Distribution. Journal of Applied Statistics. 1987;36:358-369.

42. Owoloko EA, Oguntunde PE, Adejumo AO. Performance rating of the transmuted exponential distribution: an analytical approach. Springer plus. 2015;4:818. 